\title{
MARKOVIAN PROPERTIES OF THE SPIN-BOSON MODEL
}

\author{
Ameur DHAHRI \\ Institut C. Jordan \\ Université C. Bernard, Lyon 1 \\ 21, av Claude Bernard \\ 69622 Villeurbanne Cedex \\ France \\ e-mail: dhahri@math.univ-lyon1.fr
}

\begin{abstract}
We systematically compare the Hamiltonian and Markovian approaches of quantum open system theory, in the case of the spin-boson model. We first give a complete proof of the weak coupling limit and we compute the Lindblad generator of this model. We study properties of the associated quantum master equation such as decoherence, detailed quantum balance and return to equilibrium at inverse temperature $0<\beta \leq \infty$. We further study the associated quantum Langevin equation, its associated interaction Hamiltonian. We finally give a quantum repeated interaction model describing the spin-boson system where the associated Markovian properties are satisfied without using any assumption.
\end{abstract}

\section{Introduction}

In the quantum theory of irreversible evolutions two different approaches have usually been considered by physists as well as mathematicians: The Hamiltonian and the Markovian approaches.

The Hamiltonian approach consists in giving a full Hamiltonian model for the interaction of a simple quantum system with a quantum field (particle gas, heat bath...) and to study the ergodic properties of the associated quantum dynamical system. The usual tools are then typically: modular theory of von Neumann algebras, KMS states...(cf [BR], [JP1], [JP2]...).

The Markovian approach consists in giving up the idea of modelizing the environment and concentrating on the effective dynamic of the small system. This dynamic is supposed to be described by a (completely positive) semigroup and the studies concentrate on its Lindblad generator, or on the associated quantum Langevin equation (cf [F1]-[F3], [FR1], [FR2], [P], [HP]).

In this article we systematically compare the two approaches in the case of the wellknown spin-boson model. The first step in relating the Hamiltonian and Markovian models is to derive the Lindblad generator from the Hamiltonian description, by means 
of the weak coupling limit. We indeed give a complete proof of the convergence of the Hamiltonian evolution to a Lindblad semigroup in the van Hove limit. We derive an explicit form for the generator in terms of Hamiltonian, this is treated in section 3.

In section 4, 5 and 6 we study the basic properties of the quantum master equation associated to the Lindbladian obtained in section 3. We investigate the quantum decoherence property, we show that the quantum detailed balance condition is satisfied with respect to the thermodynamical equilibrium state of the spin system and we prove the convergence to equilibrium in all cases.

In section 7 we consider the natural quantum Langevin equation associated to our Lindblad generator. We indeed, introduce a natural unitary ampliation of the quantum master equation in terms of a Schrodinger equation perturbed by quantum noises. Such a quantum Langevin equation is actually a unitary evolution in the interaction picture, we compute the associated Hamiltonian which we compare to the initial Hamiltonian.

Finally we give a quantum repeated interaction model which allows to prove that the Markovian properties of the spin-boson system are satisfied without assuming any hypothesis.

\section{The model}

\section{$2.1 \quad$ Spin-boson system}

The model we shall consider all along this article is the spin-boson model, that is, a two level atom interacting with a reservoir modelized by a free bose gas at thermal equilibrium for the temperature $T=\frac{1}{k \beta}$ (the case of zero temperature, i.e $\beta=\infty$, is also treated). Let us start by defining the spin-boson system at positive temperature. We first introduce the isolated spin and the free reservoir, and we describe the coupled system.

The Hilbert space of the isolated spin is $\mathcal{K}=\mathbb{C}^{2}$ and its Hamiltonian is $h_{S}=\sigma_{z}$, where

$$
\sigma_{z}=\left(\begin{array}{cc}
1 & 0 \\
0 & -1
\end{array}\right)
$$

The associated eigenenergies are $e_{ \pm}= \pm 1$ and we denote the corresponding eigenstates by $\Psi_{ \pm}$. The algebra of observables of the spin is $M_{2}$, the algebra of all complex $2 \times 2$ matrix. At inverse temperature $\beta$, the equilibrium state of the spin is the normal state defined by the Gibbs Ansatz

$$
\omega_{S}(A)=\frac{1}{Z} \operatorname{Tr}\left(\exp \left(-\beta \sigma_{z}\right) A\right), \text { for all } A \in M_{2},
$$

where $Z=\operatorname{Tr}\left(\exp \left(-\beta \sigma_{z}\right)\right)$.

The dynamics of the spin is defined as

$$
\tau_{S}^{t}(A)=e^{i t \sigma_{z}} A e^{-i t \sigma_{z}}, \text { for all } A \in M_{2}, t \in \mathbb{R} .
$$

The free reservoir is modelized by a free bose gas which is described by the symmetric Fock space $\Gamma_{s}\left(L^{2}\left(\mathbb{R}^{3}\right)\right)$. If we set $\omega(k)=|k|$ the energy of a single boson with 
momentum $k \in \mathbb{R}^{3}$, then the Hamiltonian of the reservoir is given by the second differential quantization $d \Gamma(\omega)$ of $\omega$. In terms of the usual creation and annihilation operators $a^{*}(k), a(k)$, we have

$$
d \Gamma(\omega)=\int_{\mathbb{R}^{3}} \omega(k) a^{*}(k) a(k) d k .
$$

The Weyl's operator associated to an element $f \in L^{2}\left(\mathbb{R}^{3}\right)$ is the operator

$$
W(f)=\exp (i \varphi(f))
$$

where $\varphi(f)$ is the self-adjoint field operator defined by

$$
\varphi(f)=\frac{1}{\sqrt{2}} \int_{\mathbb{R}^{3}}\left(a(k) \bar{f}(k)+a^{*}(k) f(k)\right) d k .
$$

Let $\mathcal{D}_{l o c}$ the space of $f \in L^{2}(\mathbb{R})$ whose Fourier transform is compactly supported. It follows from [JP2] that the Weyl's algebra, $\mathcal{A}_{l o c}=W\left(\mathcal{D}_{l o c}\right)$, the algebra generated by the set $\left\{W(f), f \in \mathcal{D}_{l o c}\right\}$ is a natural minimal set of observables associated to the reservoir. The equilibrium state of the reservoir at inverse temperature $\beta$ is given by

$$
\omega_{R}(W(f))=\exp \left[-\frac{\|f\|^{2}}{4}-\frac{1}{2} \int_{\mathbb{R}^{3}}|f(k)|^{2} \rho(k) d k\right]
$$

where $\rho(k)$ is related to $\omega(k)$ by Planck's radiation law

$$
\rho(k)=\frac{1}{e^{\beta \omega(k)}-1} .
$$

The dynamics of the reservoir is generated by $H_{b}=[d \Gamma(\omega),$.$] and it induces a Bogoliubov$ transformation

$$
\exp (i t d \Gamma(\omega)) W(f) \exp (-i t d \Gamma(\omega))=W(\exp (-i \omega t) f)
$$

The coupled system is described by the $\mathbb{C}^{*}$-algebra $M_{2} \otimes \mathcal{A}_{\text {loc }}$. The free dynamics is given by

$$
\tau_{0}^{t}(A)=\tau_{S}^{t} \otimes \tau_{R}^{t}(A), \text { for all } A \in M_{2} \otimes \mathcal{A}_{l o c} .
$$

\subsection{Semistandard representation}

The semistandard representation of the coupled system (reservoir+spin) is the representation which is standard on its reservoir part, but not standard on the spin part (cf $[\mathrm{DF}])$. Now, let us introduce the Araki-Woods representation of the couple $\left(\omega_{R}, \mathcal{A}_{\text {loc }}\right)$ which is the triple $\left(\mathcal{H}_{R}, \pi_{R}, \Omega_{R}\right)$, defined by

- $\mathcal{H}_{R}=l^{2}\left(\Gamma_{s}\left(L^{2}\left(\mathbb{R}^{3}\right)\right)\right.$, the space of Hilbert-Schmidt on $\Gamma_{s}\left(L^{2}\left(\mathbb{R}^{3}\right)\right)$ which is naturally identified as $\Gamma_{s}\left(L^{2}\left(\mathbb{R}^{3}\right)\right) \otimes \overline{\Gamma_{s}\left(L^{2}\left(\mathbb{R}^{3}\right)\right)}$ and equipped with the scalar product $(X, Y)=\operatorname{Tr}\left(X^{*} Y\right)$,

- $\pi_{R}(W(f)): X \longmapsto W\left((1+\rho)^{1 / 2} f\right) X W\left(\rho^{1 / 2} \bar{f}\right)$ for all $X \in \mathcal{H}_{R}$, 
- $\Omega_{R}=|\Omega><\Omega|$, where $\Omega$ is the vacuum vector of $\Gamma_{s}\left(L^{2}\left(\mathbb{R}^{3}\right)\right)$.

Moreover a straightforward computation show that

$$
\omega_{R}(A)=\left(\Omega_{R}, \pi_{R}(A) \Omega_{R}\right),
$$

and the relation

$$
\pi_{R}(\exp (i t d \Gamma(\omega)) A \exp (-i t d \Gamma(\omega)))=\exp (i t[d \Gamma(\omega), .]) \pi_{R}(A) \exp (-i t[d \Gamma(\omega), .])
$$

defines a dynamics on $\mathcal{M}_{R}=\pi_{R}\left(\mathcal{A}_{\text {loc }}\right)^{\prime \prime}$ whose generator is the operator

$$
L_{R}=[d \Gamma(\omega), .] .
$$

The free semi-Liouvillean associated to the semistandard representation of the spinboson system is defined by

$$
L_{0}^{s e m i}=\sigma_{z} \otimes 1+1 \otimes L_{R}
$$

The full semi-Liouvillean is the operator

$$
L_{\lambda}^{s e m i}=L_{0}^{s e m i}+\lambda \sigma_{x} \otimes \varphi_{A W}(\alpha),
$$

where $\lambda \in \mathbb{R}$, and where $\alpha \in L^{2}\left(\mathbb{R}^{3}\right)$ is called the test function (or cut-off function), $\varphi_{A W}(\alpha)$ is the field operator of the Araki-Woods representation which can be identified as follows

$$
\varphi_{A W}(\alpha) \simeq \varphi\left((1+\rho)^{1 / 2} \alpha\right) \otimes 1+1 \otimes \varphi\left(\bar{\rho}^{1 / 2} \bar{\alpha}\right)
$$

(see [JP2], [DJ] for more details) and

$$
\sigma_{x}=\left(\begin{array}{cc}
0 & 1 \\
1 & 0
\end{array}\right)
$$

The following proposition follows from [JP2].

Proposition 2.1 If $\left(\omega+\omega^{-1}\right) \alpha \in L^{2}\left(\mathbb{R}^{3}\right)$ then $L_{\lambda}^{\text {semi }}$ is essentially self-adjoint operator on $\mathbb{C}^{2} \otimes D(d \Gamma(\omega)) \otimes D(d \Gamma(\omega))$ for all $\lambda \in \mathbb{R}$.

An immediate consequence of the above proposition is that

$$
\tau_{\lambda}^{t}(A)=e^{i t L_{\lambda}^{s e m i}} A e^{-i t L_{\lambda}^{s e m i}}
$$

defines a dynamics on $\mathcal{M}=M_{2} \otimes \mathcal{M}_{R}$. 


\subsection{Gluing of reservoir 1-particle spaces}

After taking the Araki-Woods representation of the pair $\left(\omega_{R}, \mathcal{A}_{l o c}\right)$, we distinguish that the reservoir state is non-Fock (i.e: it cannot be represented as a pure state on a Fock space) and this case is more complicated to treat. By using the gluing (see [DJ], [JP1]), we see that this state can be represented as a pure state on a Fock space. Hence we have

$$
\begin{aligned}
& -\Gamma_{s}\left(L^{2}\left(\mathbb{R}^{3}\right)\right) \otimes \overline{\Gamma_{s}\left(L^{2}\left(\mathbb{R}^{3}\right)\right)} \simeq \Gamma_{s}\left(L^{2}\left(\mathbb{R}^{3}\right)\right) \otimes \Gamma_{s}\left(\overline{L^{2}\left(\mathbb{R}^{3}\right)}\right) \simeq \Gamma_{s}\left(L^{2}\left(\mathbb{R}^{3}\right) \oplus \overline{L^{2}\left(\mathbb{R}^{3}\right)}\right), \\
& -L_{R} \simeq d \Gamma(\omega \oplus-\bar{\omega}), \\
& \text { - } \varphi_{A W}(\alpha) \simeq \varphi\left((1+\rho)^{1 / 2} \alpha \oplus \bar{\rho}^{1 / 2} \bar{\alpha}\right) . \\
& \text { - } \Omega_{R} \simeq \Omega \oplus \bar{\Omega} .
\end{aligned}
$$

Therefore, it is obvious that $\omega_{R}$ is a pure state which is defined on the Fock space $\Gamma_{s}\left(L^{2}\left(\mathbb{R}^{3}\right) \oplus \overline{L^{2}\left(\mathbb{R}^{3}\right)}\right)$. Moreover the Bogoliubov transformation is satisfied and we have

$$
e^{i t d \Gamma(\omega \oplus-\bar{\omega})} \varphi_{A W}(\alpha) e^{-i t d \Gamma(\omega \oplus-\bar{\omega})}=\varphi_{A W}\left(e^{-i t \omega} \alpha\right) .
$$

This simplifies our formulation.

\section{Weak coupling limit of the spin-boson system}

\subsection{Abstract theory}

Let us recall some elements of the general theory of weak coupling limit. Let $\mathcal{X}$ be a Banach space, $P$ a projection on $\mathcal{X}$. Let $e^{i t L_{0}}$ be a one-parameter group of isometries commuting with $P$. We put $E=P L_{0}$. Clearly $E$ is the generator of a one-parameter group of isometries on $\operatorname{Ran} P$.

Let $Q$ be a perturbation of the operator $L_{0}$ with domain $\operatorname{Dom} Q \supset \operatorname{Dom} L_{0}$ and we suppose that $L_{\lambda}=L_{0}+\lambda Q$ generates a one-parameter semigroup of isometries. Besides we assume that $P Q P, P Q(1-P)$ and $(1-P) Q P$ are bounded.

Set

$$
K_{\lambda}(t)=i \int_{0}^{\lambda^{-2} t} e^{-i s(E+\lambda P Q P)} P Q e^{i s(1-P) L_{\lambda}(1-P)} Q P d s .
$$

The following two theorems are proved in $[\mathrm{DF}]$.

Theorem 3.1 Suppose the following assumptions are satisfied

(1) For all $t_{1}>0$ there exists $c$ such that

$$
\sup _{|\lambda|<1} \sup _{0 \leq t \leq t_{1}}\left\|K_{\lambda}(t)\right\| \leq c
$$


(2) There exists an operator $K$ on RanP such that

$$
\lim _{\lambda \rightarrow 0} K_{\lambda}=K
$$

for all $0<t<\infty$.

Then

$$
\lim _{\lambda \rightarrow 0} \sup _{0 \leq t \leq t_{1}}\left\|P e^{i t L_{\lambda} / \lambda^{2}} P-e^{i t\left(E+\lambda P Q P+\lambda^{2} K\right) / \lambda^{2}}\right\|=0 .
$$

Set

$$
K^{\sharp}=s-\lim _{t \rightarrow \infty} \frac{1}{t} \int_{0}^{t} e^{i s E} K e^{-i s E} d s .
$$

Theorem 3.2 If in addition to assumptions of Theorem 3.1 we have that $P Q P=0$ and $K^{\sharp}$ exists, then

$$
\lim _{\lambda \rightarrow 0} \sup _{0 \leq t \leq t_{0}}\left\|e^{-i t E / \lambda^{2}} P e^{i t\left(L_{0}+\lambda Q\right) / \lambda^{2}} P-e^{i t K^{\sharp}}\right\|=0 .
$$

Remark 1: If $\operatorname{Ran} P$ is a finite dimensional space then $K^{\sharp}$ is given by

$$
K^{\sharp}=\sum_{e \in s p E} \mathbb{I}_{e}(E) K \mathbb{1}_{e}(E) .
$$

\subsection{Application to the spin-boson system}

Recall that in the semistandard representation of the spin-boson system, the free semiLiouvillean is the operator

$$
L_{0}^{s e m i}=\sigma_{z} \otimes 1+1 \otimes L_{R},
$$

and the full semi-Liouvillean is given by

$$
L_{\lambda}^{s e m i}=L_{0}^{s e m i}+\lambda \sigma_{x} \otimes \varphi_{A W}(\alpha) .
$$

Set $V=\sigma_{x} \otimes \varphi_{A W}(\alpha)$. We put

$$
\delta_{\lambda}=\left[L_{\lambda}^{s e m i}, .\right]=\delta_{0}+\lambda[V, .],
$$

with $\delta_{0}=\left[L_{0}^{s e m i},.\right]$, the generator of the dynamics $\tau_{\lambda}^{t}$. For $B \otimes C \in \mathcal{M}$, we define the projection $P$ by

$$
P(B \otimes C)=\omega_{R}(C) B \otimes 1_{\mathcal{H}_{R}} .
$$

In particular we have

$$
E=P \delta_{0}=\delta_{0} P=\left[\sigma_{z}, .\right] P \text { and } P[V, .] P=0 .
$$

Set $P_{1}=1-P$.Then it follows that

$$
K_{\lambda}(t)=i \int_{0}^{\lambda^{-2} t} e^{-i s E} P[V, .] e^{i s P_{1}\left[L_{\lambda}^{s e m i}, .\right] P_{1}}[V, .] P d s .
$$


Note that $P[V,] P=0,. P_{1}$ commutes with $\left[L_{0}^{\text {semi }},.\right]$ and

$$
e^{i s P_{1}\left[L_{0}^{s e m i}, .\right] P_{1}}=e^{i s\left[L_{0}^{s e m i}, \cdot\right]} P_{1}+P .
$$

Thus, if we suppose that

$$
K=i \int_{0}^{\infty} e^{-i s E} P[V, .] e^{i s P_{1}\left[L_{0}^{s e m i}, .\right] P_{1}}[V, .] P d s
$$

exists, we have

$$
K=i \int_{0}^{\infty} e^{-i s E} P[V, .] e^{i s\left[L_{0}^{s e m i}, .\right]}[V, .] P d s .
$$

In the following we assume that $\left(\omega+\omega^{-1}\right) \alpha \in L^{2}\left(\mathbb{R}^{3}\right)$ and we propose to show, under some conditions, that $K$ exists and the operator $K_{\lambda}$ converges to $K$ when $\lambda \longrightarrow 0$. Set

$$
U_{t}^{\lambda}=e^{i t P_{1}\left[L_{\lambda}^{\text {semi }}, .\right] P_{1}}, \quad U_{t}=e^{i t P_{1}\left[L_{0}^{\text {semi }}, .\right] P_{1}} .
$$

We thus have

$$
U_{t}^{\lambda}=U_{t}+i \lambda \int_{0}^{t} U_{t-s} P_{1}[V, .] P_{1} U_{s}^{\lambda} d s
$$

Hence the operator $U_{-t} U_{t}^{\lambda}$ satisfies the equation

$$
U_{-t} U_{t}^{\lambda}=1+i \lambda \int_{0}^{t}\left(U_{-s} P_{1}[V, .] P_{1} U_{s}\right)\left(U_{-s} U_{s}^{\lambda}\right) d s .
$$

Therefore we get the following series of iterated integrals

$$
U_{-t} U_{t}^{\lambda}=1+\sum_{n \geq 1}(i \lambda)^{n} \int_{0 \leq t_{n} \leq \ldots \leq t_{1} \leq t}\left(U_{-t_{1}} P_{1}[V, .] P_{1} U_{t_{1}}\right) \ldots\left(U_{-t_{n}} P_{1}[V, .] P_{1} U_{t_{n}}\right) d t_{n} \ldots d t_{1}
$$

Note that the operator $U_{t_{k}}$ commutes with $P_{1}$. So, if we put

$$
Q_{k}=U_{-t_{k}}[V, .] U_{t_{k}}
$$

then

$$
U_{-t} U_{t}^{\lambda}=1+\sum_{n \geq 1}(i \lambda)^{n} \int_{0 \leq t_{n} \leq \ldots \leq t_{1} \leq t}\left(P_{1} Q_{1} P_{1}\right) \ldots\left(P_{1} Q_{n} P_{1}\right) d t_{n} \ldots d t_{1}
$$

and

$$
\begin{aligned}
& K_{\lambda}(t)=i \int_{0}^{\lambda^{-2} t} e^{-i s E} P[V, .] e^{i s P_{1}\left[L_{0}^{\text {semi }}, .\right] P_{1}}[V, .] P d s+ \\
& i \sum_{n \geq 1}(i \lambda)^{n} \int_{0 \leq t_{n} \leq \ldots \leq t_{0} \leq \lambda^{-2} t} e^{-i t_{0} E} P[V, .] U_{t_{0}}\left(P_{1} Q_{1} P_{1}\right) \ldots .\left(P_{1} Q_{n} P_{1}\right)[V, .] P d t_{n} \ldots d t_{0} .(4)
\end{aligned}
$$

Let us put

$$
R_{n}(t)=\int_{0 \leq t_{n} \leq \ldots \leq t_{0} \leq t} e^{-i t_{0} E} P[V, .] U_{t_{0}}\left(P_{1} Q_{1} P_{1}\right) \ldots .\left(P_{1} Q_{n} P_{1}\right)[V, .] P d t_{n} \ldots d t_{0} .
$$


Recall that $P U_{-t_{0}}=P$, hence if we set $Q_{n+1}=U_{-t_{n+1}}[V,.] U_{t_{n+1}}$, with $t_{n+1}=0$, we get

$$
R_{n}(t)=\int_{0 \leq t_{n} \leq \ldots \leq t_{0} \leq t} e^{-i t_{0} E} P Q_{0}\left(P_{1} Q_{1} P_{1}\right) \ldots\left(P_{1} Q_{n} P_{1}\right) Q_{n+1} P d t_{n} \ldots d t_{0} .
$$

\section{Lemma 3.3}

$R_{n}(t)=\int_{0 \leq t_{n} \leq \ldots \leq t_{0} \leq t} P\left[\sigma_{x, 0} \otimes \varphi_{A W}\left(e^{-i t_{0} \omega} \alpha\right),.\right] P_{1} \ldots P_{1}\left[\sigma_{x, n+1} \otimes \varphi_{A W}\left(e^{-i t_{n+1} \omega}\right),.\right] P d t_{n} \ldots d t_{0}$, where $t_{n+1}=0, \sigma_{x, r}=e^{-i t_{r} \sigma_{z}} \sigma_{x} e^{i t_{r} \sigma_{z}}$.

\section{Proof}

Let us start by computing $P_{1} Q_{r} P_{1}$ for $r \geq 1$. We have

$$
U_{t_{r}}=e^{i t_{r}\left[\sigma_{z}, .\right]} e^{i t_{r}\left[L_{R}, \cdot\right]} P_{1}+P,
$$

and

$$
U_{t_{r}} P_{1}=e^{i t_{r}\left[\sigma_{z}, \cdot\right]} e^{i t_{r}\left[L_{R}, \cdot\right]} P_{1}
$$

Therefore it follows that

$$
P_{1} U_{-t_{r}}[V, .] U_{t_{r}} P_{1}=P_{1} e^{-i t_{r}\left[\sigma_{z}, .\right]} e^{-i t_{r}\left[L_{R}, \cdot\right]}[V, .] e^{i t_{r}\left[\sigma_{z}, .\right]} e^{i t_{r}\left[L_{R}, .\right]} P_{1} .
$$

Furthermore we have

$$
e^{-i t_{r}\left[\sigma_{z},\right]} e^{-i t_{r}\left[L_{R}, \cdot\right]}[V, .] e^{i t_{r}\left[\sigma_{z}, .\right]} e^{i t_{r}\left[L_{R}, .\right]}(B \otimes C)=\left[\sigma_{x, r} \otimes e^{-i t_{r} L_{R}} \varphi_{A W}(\alpha) e^{i t_{r} L_{R}}, .\right](B \otimes C),
$$

and

$$
e^{-i t_{r} L_{R}} \varphi_{A W}(\alpha) e^{i t_{r} L_{R}}=\varphi_{A W}\left(e^{-i t_{r} \omega} \alpha\right)
$$

This gives

$$
P_{1} Q_{r} P_{1}=P_{1}\left[\sigma_{x, r} \otimes \varphi_{A W}\left(e^{-i t_{r} \omega} \alpha\right), .\right] P_{1} .
$$

Besides, $P e^{-t_{0}\left[\sigma_{z}, .\right]}=P e^{-i t_{0}\left[\sigma_{z}, .\right]} e^{-i t_{0}\left[L_{R}, \cdot\right]}$ and

$$
\begin{aligned}
e^{-i t_{0} E} P Q_{0} P_{1} & =P e^{-i t_{0}\left[\sigma_{z}, .\right]}[V, .] e^{i t_{0}\left[\sigma_{z}, .\right]} e^{i t_{0}\left[L_{R}, .\right]} P \\
& =P e^{-i t_{0}\left[\sigma_{z}, .\right]} e^{-i t_{0}\left[L_{R}, \cdot\right]}[V, .] e^{i t_{0}\left[\sigma_{z}, \cdot\right]} e^{i t_{0}\left[L_{R}, \cdot\right]} P \\
& =P\left[\sigma_{x, 0} \otimes \varphi_{A W}\left(e^{-i t_{0} \omega} \alpha\right), .\right] P_{1} .
\end{aligned}
$$

Thus from relation (6), the lemma holds.

\section{Lemma 3.4}

$$
R_{2 n+1}(t)=0 \text {. }
$$

\section{Proof}

Note that

$$
\begin{array}{r}
P\left[\sigma_{x, 0} \otimes \varphi_{A W}\left(e^{-i t_{0} \omega} \alpha\right), .\right] P_{1} \ldots P_{1}\left[\sigma_{x, 2 n+2} \otimes \varphi_{A W}\left(e^{-i t_{2 n+2} \omega} \alpha\right), .\right] P= \\
P\left[\sigma_{x, 0} \otimes \varphi_{A W}\left(e^{-i t_{0} \omega} \alpha\right), .\right](1-P)\left[\sigma_{x, 1} \otimes \varphi_{A W}\left(e^{-i t_{1} \omega} \alpha\right), .\right](1-P) \ldots \\
\ldots(1-P)\left[\sigma_{x, 2 n+2} \otimes \varphi_{A W}\left(e^{-i t_{2 n+2} \omega} \alpha\right), .\right] P .
\end{array}
$$


Therefore, if we expand the right-hand side of equation (7), then we get a sum of terms each of which is a product of elements of the form

$$
P\left[\sigma_{x, p_{k}} \otimes \varphi_{A W}\left(e^{-i t_{p_{k}} \omega} \alpha\right), .\right] \ldots\left[\sigma_{x, p_{m}} \otimes \varphi_{A W}\left(e^{-i t_{p_{m}} \omega} \alpha\right), .\right] P,
$$

where $0 \leq p_{k} \leq \ldots \leq p_{m} \leq \ldots \leq 2 n+2$. But, in each product there exists at least an element of the form

$$
P\left[\sigma_{x, r_{1}} \otimes \varphi_{A W}\left(e^{-i t_{r_{1}} \omega} \alpha\right), .\right] \ldots\left[\sigma_{x, r_{2 p+1}} \otimes \varphi_{A W}\left(e^{-i t_{r_{2 p+1}} \omega} \alpha\right), .\right] P
$$

where $0 \leq r_{1} \leq \ldots \leq r_{2 p+1} \leq \ldots \leq r_{2 n+2}$. Furthermore, it is easy to show that

$$
\left[\sigma_{x, r_{1}} \otimes \varphi_{A W}\left(e^{-i t_{r_{1}} \omega} \alpha\right), .\right] \ldots\left[\sigma_{x, r_{2 p+1}} \otimes \varphi_{A W}\left(e^{-i t_{r_{2 p+1}} \omega} \alpha\right), .\right] P(B \otimes C)
$$

is a sum of terms each of which has a second component composed by $2 p+1$ number product of vector fields. But the projection $P$ acts uniquely in the second component and the Gibbs state $\omega_{R}$ of the reservoir is a quasi-free state (see $[\mathrm{BR}]$ ). Then it follows that

$$
P\left[\sigma_{x, r_{1}} \otimes \varphi_{A W}\left(e^{-i t r_{1} \omega} \alpha\right), .\right] \ldots\left[\sigma_{x, r_{2 p+1}} \otimes \varphi_{A W}\left(e^{-i t_{r_{2 p+1}} \omega} \alpha\right), .\right] P(B \otimes C)=0,
$$

and by Lemma $3.3, R_{2 n+1}(t)=0$.

Remark 2: By the proof of Lemma 3.4 we can deduce that $R_{2 n}(t)$ is a sum of $2^{n}$ terms each of which is a product containing only an even number of products of commutators of the form $\left[\sigma_{x, r} \otimes \varphi_{A W}\left(e^{-i t_{r} \omega} \alpha\right),.\right]$ between two successive projections $P$.

Theorem 3.5 Suppose that the following assumptions hold

(i) $\left\|R_{2 n}(t)\right\| \leq c_{n} t^{n}$, where the series $\sum_{n \geq 1} c_{n} t^{n}$ has infinite radius of convergence.

(ii) There exists $0<\varepsilon<1$ and a sequence $d_{n} \geq 0$ such that

$$
\left\|R_{2 n}(t)\right\| \leq d_{n} t^{n-\epsilon}
$$

Then

$$
\lim _{\lambda \rightarrow 0} \sum_{n \geq 1}(i \lambda)^{n} R_{2 n}\left(\lambda^{-2} t\right)=0
$$

\section{Proof}

The proof of this theorem is a straightforward application of Lebesgue's Theorem.

Now the aim is to introduce some conditions which ensures that assumptions (i) and (ii) of the above theorem are satisfied. Set

$$
h(t)=<e^{-i t L_{R}} \varphi_{A W}(\alpha) e^{i t L_{R}} \varphi_{A W}(\alpha) \Omega_{R}, \Omega_{R}>.
$$

Let us recall that

$$
L_{R}=[d \Gamma(\omega), .] \simeq d \Gamma(\omega \oplus-\bar{\omega}),
$$


and

$$
e^{-i t L_{R}} \varphi_{A W}(\alpha) e^{i t L_{R}}=\varphi_{A W}\left(e^{-i t \omega} \alpha\right)
$$

Therefore we get

$$
h(t)=<\varphi_{A W}\left(e^{-i t \omega} \alpha\right) \varphi_{A W}(\alpha) \Omega_{R}, \Omega_{R}>.
$$

Moreover, a straightforward computation shows that

$$
h(t-s)=<\varphi_{A W}\left(e^{-i t \omega} \alpha\right) \varphi_{A W}\left(e^{-i s \omega} \alpha\right) \Omega_{R}, \Omega_{R}>.
$$

Now, for any integer $n$ we define the set $\mathcal{P}_{n}$ of pairings as the set of permutations $\sigma$ of $(1, \ldots, 2 n)$ such that

$$
\sigma(2 r-1)<\sigma(2 r) \text { and } \sigma(2 r-1)<\sigma(2 r+1)
$$

for all $r$. Let us put

$$
\begin{aligned}
<\varphi_{A W}\left(\alpha_{1}\right) \ldots \varphi_{A W}\left(\alpha_{n}\right)> & =\omega_{R}\left(\varphi_{A W}\left(\alpha_{1}\right) \ldots \varphi_{A W}\left(\alpha_{n}\right)\right) \\
& =<\Omega_{R}, \varphi_{A W}\left(\alpha_{1}\right) \ldots \varphi_{A W}\left(\alpha_{n}\right) \Omega_{R}>
\end{aligned}
$$

So, if $n=2$ then $\left\langle\varphi_{A W}\left(\alpha_{1}\right) \varphi_{A W}\left(\alpha_{2}\right)>\right.$ is called the two point correlations matrix. Furthermore we have

$$
<\varphi_{A W}\left(\alpha_{1}\right) \ldots \varphi_{A W}\left(\alpha_{2 n}\right)>=\sum_{\sigma \in \mathcal{P}_{n}} \prod_{r=1}^{n}<\varphi_{A W}\left(\alpha_{\sigma(2 r-1)}\right) \varphi_{A W}\left(\alpha_{\sigma(2 r)}\right)>,
$$

and

$$
<\varphi_{A W}\left(\alpha_{1}\right) \ldots \varphi_{A W}\left(\alpha_{2 n+1}\right)>=0 .
$$

(see $[\mathrm{BR}] \mathrm{P} 40$ for more details).

The proof of the following lemma is similar as the one of Lemma 3.3 in [D1].

Lemma 3.6 If $\|h\|_{1} \leq \infty$ then for any permutation $\pi$ of $(0,1, \ldots, 2 n+1)$ we have

$$
\left|\sum_{\sigma \in \mathcal{P}_{(0,1, \ldots, 2 n+1)}} \int_{0 \leq t_{2 n} \leq \ldots \leq t_{0} \leq t} \prod_{r=0}^{n} h\left(t_{\pi \sigma(2 r)}-t_{\pi \sigma(2 r+1)}\right) d t_{2 n} \ldots d t_{0}\right| \leq \frac{1}{2^{n+1}(n+1) !}\|h\|_{1}^{n+1} t^{n},
$$

with $t_{2 n+1}=0$.

We now prove the following.

Theorem 3.7 If $\|h\|_{1} \leq \infty$ then

$$
\left\|R_{2 n}(t)\right\| \leq 2^{2 n+1}\|h\|_{1}^{n+1} \frac{t^{n}}{(n+1) !} .
$$

\section{Proof}

Let us put

$$
\Phi_{r}=\varphi_{A W}\left(e^{-i t_{r} \omega} \alpha\right), \Phi_{r}^{L} C=\Phi_{r} C, \Phi_{r}^{R} C=C \Phi_{r},
$$


$\sigma_{x, r}^{L} B=\sigma_{x, r} B, \quad \sigma_{x, r}^{R} B=B \sigma_{x, r}$,

$\beta$ : a function from $\{0,1, \ldots, 2 n+1\}$ to $\{L, R\}$,

$k_{\beta}=\sharp\{r \in\{0,1, \ldots, 2 n+1\}$ such that $\beta(r)=R\}$.

In the sequel, we simplify the notation $\sigma_{x, r} \otimes \Phi_{r}$ into $\sigma_{x, r} \Phi_{r}$. With this notations we have

$$
\left[\sigma_{x, r} \Phi_{r}, .\right]=\sigma_{x, r}^{L} \Phi_{r}^{L}-\sigma_{x, r}^{R} \Phi_{r}^{R}
$$

Let us recall that, from remark 2 and Lemma 3.3, $R_{2 n}(t)$ is a sum of $2^{n}$ terms each of which is of the form

$$
\begin{array}{r}
C_{2 n, j}(t)=(-1)^{j} \int_{0 \leq t_{2 n} \leq \ldots \leq t_{0} \leq t} \sum_{\beta}(-1)^{k_{\beta}} P\left(\sigma_{x, 0}^{\beta(0)} \Phi_{0}^{\beta(0)}\right)\left(\sigma_{x, 1}^{\beta(1)} \Phi_{1}^{\beta(1)}\right) \ldots \\
\ldots\left(\sigma_{x, p_{1}-1}^{\beta\left(p_{1}-1\right)} \Phi_{p_{1}-1}^{\beta\left(p_{1}-1\right)}\right) P\left(\sigma_{x, p_{1}}^{\beta\left(p_{1}\right)} \Phi_{p_{1}}^{\beta\left(p_{1}\right)}\right) \ldots\left(\sigma_{x, p_{j}-1}^{\beta\left(p_{j}-1\right)} \Phi_{p_{j}-1}^{\beta\left(p_{j}-1\right)}\right) \times \\
P\left(\sigma_{x, p_{j}}^{p_{j}} \Phi_{p_{j}}^{\beta\left(p_{j}\right)}\right) \ldots\left(\sigma_{x, 2 n}^{\beta(2 n)} \Phi_{2 n}^{\beta(2 n)}\right)\left(\sigma_{x, 2 n+1}^{\beta(2 n+1)} \Phi_{2 n+1}^{\beta(2 n+1)}\right) P d t_{2 n} \ldots d t_{0},
\end{array}
$$

where $0=p_{0}<p_{1}<p_{2}<\ldots<p_{j}<p_{j+1}=2 n+2$, each $p_{j}$ is an even number and $j=N-2$, with $N$ is the number of projections $P$ which appears in the expression of $C_{2 n, j}(t)$.

Hence we have

$$
\begin{aligned}
\left\|C_{2 n, j}(t)(B \otimes C)\right\| & \leq\|B \otimes C\| \sum_{\beta} \int_{0 \leq t_{2 n} \leq \ldots \leq t_{0} \leq t} \prod_{r=0}^{j}\left|\omega_{R}\left(\Phi_{p_{r}}^{\beta\left(p_{r}\right)} \ldots \Phi_{p_{r+1}-1}^{\beta\left(p_{r+1}-1\right)}\right)\right| d t_{2 n} \ldots d t_{0}, \\
& \leq\|B \otimes C\| \sum_{\beta} \int_{0 \leq t_{2 n} \leq \ldots \leq t_{0} \leq t} \prod_{r=0}^{j}\left|<\Phi_{p_{r}}^{\beta\left(p_{r}\right)} \ldots \Phi_{p_{r+1}-1}^{\beta\left(p_{r+1}-1\right)}>\right| d t_{2 n} \ldots d t_{0}, \\
& \leq\|B \otimes C\| \sum_{\beta} \int_{\leq t_{2 n} \leq \ldots \leq t_{0} \leq t} \prod_{r=0}^{j}\left|<\Phi_{\pi\left(p_{r}\right)} \ldots \Phi_{\pi\left(p_{r+1}-1\right)}>\right| d t_{2 n} \ldots d t_{0},
\end{aligned}
$$

where $\pi$ is a permutation which depends on $\beta$.

Thus from equation (10) and Lemma 3.6 we get

$$
\begin{aligned}
\left\|C_{2 n, j}(t)\right\| & \leq \sum_{\beta} \sum_{\sigma \in \mathcal{P}_{(0,1, \ldots, 2 n+1)}} \int_{0 \leq t_{2 n} \leq \ldots \leq t_{0} \leq t} \prod_{r=0}^{n}\left|<\Phi_{\pi(\sigma(2 r))} \Phi_{\pi(\sigma(2 r+1))}>\right| d t_{2 n} \ldots d t_{0} \\
& \leq 2^{2 n+2}\|h\|_{1}^{n+1} \frac{t^{n}}{2^{n+1}(n+1) !}
\end{aligned}
$$

Therefore $C_{2 n, j}$ is dominated uniformely in $j$. Finally this proves that

$$
\left\|R_{2 n}(t)\right\| \leq 2^{2 n+1}\|h\|_{1}^{n+1} \frac{t^{n}}{(n+1) !} .
$$

The following theorem ensures that assumption (ii) of Theorem 3.5 holds. 


\section{Theorem 3.8 If}

$$
\int_{0}^{\infty}\left(1+t^{\varepsilon}\right)|h(t)| d t<\infty
$$

for some $0<\varepsilon<1$, then there exists $d_{n}>0$ such that

$$
\left\|R_{2 n}(t)\right\| \leq d_{n} t^{n-\varepsilon}
$$

\section{Proof}

We have that $R_{2 n}(t)$ is a sum of $2^{n}$ terms each of which takes the form of $C_{2 n, j}$ which is defined previously. In order to prove this theorem we group those terms two by two as follows

$$
\begin{aligned}
& (-1)^{j} \int_{0 \leq t_{2 n} \leq \ldots \leq t_{0} \leq t} \sum_{\beta}(-1)^{k_{\beta}} P\left(\sigma_{x, 0}^{\beta(0)} \Phi_{0}^{\beta(0)}\right) \ldots\left(\sigma_{x, p_{1}-1}^{\beta\left(p_{1}-1\right)} \Phi_{p_{1}-1}^{\beta\left(p_{1}-1\right)}\right) P \ldots P\left(\sigma_{x, p_{j}}^{\beta\left(p_{j}\right)} \Phi_{p_{j}}^{\beta\left(p_{j}\right)}\right) \\
& \ldots\left(\sigma_{x, 2 n-1}^{\beta(2 n-1)} \Phi_{2 n-1}^{\beta(2 n-1)}\right)\left(\sigma_{x, 2 n}^{\beta(2 n)} \Phi_{2 n}^{\beta(2 n)}\right)\left(\sigma_{x, 2 n+1}^{\beta(2 n+1)} \Phi_{2 n+1}^{\beta(2 n+1)}\right) P d t_{2 n} \ldots d t_{0}+ \\
& (-1)^{(j+1)} \int_{0 \leq t_{2 n} \leq \ldots \leq t_{0} \leq t} \sum_{\beta}(-1)^{k_{\beta}} P\left(\sigma_{x, 0}^{\beta(0)} \Phi_{0}^{\beta(0)}\right) \ldots\left(\sigma_{x, p_{1}-1}^{\beta\left(p_{1}-1\right)} \Phi_{p_{1}-1}^{\beta\left(p_{1}-1\right)}\right) P \ldots P\left(\sigma_{x, p_{j}}^{\beta\left(p_{j}\right)} \Phi_{p_{j}}^{\beta\left(p_{j}\right)}\right) \\
& \ldots\left(\sigma_{x, 2 n-1}^{\beta(2 n-1)} \Phi_{2 n-1}^{\beta(2 n-1)}\right) P\left(\sigma_{x, 2 n}^{\beta(2 n)} \Phi_{2 n}^{\beta(2 n)}\right)\left(\sigma_{x, 2 n+1}^{\beta(2 n+1)} \Phi_{2 n+1}^{\beta(2 n+1)}\right) P d t_{2 n} \ldots d t_{0}= \\
& (-1)^{j} \int_{0 \leq t_{2 n} \leq \ldots \leq t_{0} \leq t} \sum_{\beta}(-1)^{k_{\beta}} P\left(\sigma_{x, 0}^{\beta(0)} \Phi_{0}^{\beta(0)}\right) \ldots\left(\sigma_{x, p_{1}-1}^{\beta\left(p_{1}-1\right)} \Phi_{p_{1}-1}^{\beta\left(p_{1}-1\right)}\right) P \ldots \\
& \ldots\left\{P\left(\sigma_{x, p_{j}}^{\beta\left(p_{j}\right)} \Phi_{p_{j}}^{\beta\left(p_{j}\right)}\right) \ldots\left(\sigma_{x, 2 n-1}^{\beta(2 n-1)} \Phi_{2 n-1}^{\beta(2 n-1)}\right)\left(\sigma_{x, 2 n}^{\beta(2 n)} \Phi_{2 n}^{\beta(2 n)}\right)\left(\sigma_{x, 2 n+1}^{\beta(2 n+1)} \Phi_{2 n+1}^{\beta(2 n+1)}\right) P-\right. \\
& \left.P\left(\sigma_{x, p_{j}}^{\beta\left(p_{j}\right)} \Phi_{p_{j}}^{\beta\left(p_{j}\right)}\right) \ldots\left(\sigma_{x, 2 n-1}^{\beta(2 n-1)} \Phi_{2 n-1}^{\beta(2 n-1)}\right) P\left(\sigma_{x, 2 n}^{\beta(2 n)} \Phi_{2 n}^{\beta(2 n)}\right)\left(\sigma_{x, 2 n+1}^{\beta(2 n+1)} \Phi_{2 n+1}^{\beta(2 n+1)}\right) P\right\} d t_{2 n} \ldots d t_{0} .
\end{aligned}
$$

Therefore the right-hand side of the above equation is dominated by

$$
\begin{gathered}
\sum_{\beta} \int_{0 \leq t_{2 n} \leq \ldots \leq t_{0} \leq t} \prod_{k=0}^{j-1}\left|<\Phi_{p_{k}}^{\beta\left(p_{k}\right)} \Phi_{p_{k}+1}^{\beta\left(p_{k}+1\right)} \ldots \Phi_{p_{k+1}-1}^{\beta\left(p_{k+1}-1\right)}>\right| \times \\
\left|\left\{<\Phi_{p_{j}}^{\beta\left(p_{j}\right)} \ldots \Phi_{2 n}^{\beta(2 n)} \Phi_{2 n+1}^{\beta(2 n+1)}>-<\Phi_{p_{j}}^{\beta\left(p_{j}\right)} \ldots \Phi_{2 n-1}^{\beta(2 n-1)}><\Phi_{2 n}^{\beta(2 n)} \Phi_{2 n+1}^{\beta(2 n+1)}>\right\}\right| d t_{2 n} \ldots d t_{0} .(11
\end{gathered}
$$

Note that in the between bracket terms, there is no product of two point correlation matrix where $2 n$ is paired with $(2 n+1)$. Moreover this term is equal to

$$
\sum_{\sigma \in \mathcal{P}_{\left(p_{j}, \ldots, 2 n+1\right)}} \prod_{r=\frac{1}{2} p_{j}}^{n}<\Phi_{\sigma(\pi(2 r))} \Phi_{\sigma(\pi(2 r+1))}>,
$$

where $2 n$ is not paired with $(2 n+1)$ and $\pi$ is a permutation which depends on $\beta$. Thus the term in equation (11) is dominated by

$$
\sum_{\sigma} \int_{0 \leq t_{2 n} \leq \ldots \leq t_{0} \leq t} \prod_{r=0}^{n}\left|<\Phi_{\sigma(2 r)} \Phi_{\sigma(2 r+1)}>\right| d t_{2 n} \ldots d t_{0},
$$


where $\sum_{\sigma}$ indicates the sum over all pairings of $\{0,1 \ldots, 2 n+1\}$ such that $2 n$ is not paired with $(2 n+1),\left(t_{2 n+1}=0\right)$.

But we have

$$
\begin{gathered}
\int_{0 \leq t_{2 n} \leq \ldots \leq t_{0} \leq t} \prod_{r=0}^{n} \mid<\Phi_{\sigma(2 r)} \Phi_{\sigma(2 r+1)}>d t_{2 n} \ldots d t_{0}= \\
\int_{0 \leq t_{2 n} \leq \ldots \leq t_{0} \leq t} \prod_{r=0}^{n}\left|h\left(t_{\sigma(2 r)}-t_{\sigma(2 r+1)}\right)\right| d t_{2 n} \ldots d t_{0} \leq \\
\operatorname{cst}\|h\|^{n} t^{k} \int_{0}^{t}|h(s)| s^{n-k} d s \leq \\
\operatorname{cst}\|h\|^{n} t^{n-\varepsilon} \int_{0}^{t}|h(s)| s^{\varepsilon} d s
\end{gathered}
$$

with $0 \leq k \leq n-1$.

This proves the theorem.

All together applying relation (4), Lemma 3.4, Theorem 3.5 to 3.8, we have proved the following.

Theorem 3.9 Suppose that the following assumptions are satisfied

(1) $\left(\omega+\omega^{-1}\right) \alpha \in L^{2}\left(\mathbb{R}^{3}\right)$,

(2) $\int_{0}^{\infty}\left(1+t^{\varepsilon}\right)|h(t)| d t<\infty$, for some $0<\varepsilon<1$,

then

$$
\lim _{\lambda \rightarrow 0}\left\|K_{\lambda}(t)-K(t)\right\|=0
$$

for all t. Moreover

$$
K^{\sharp}=i \int_{0}^{\infty} \sum_{e \in s p\left(\left[\sigma_{z}, .\right]\right)} e^{-i s e} P \mathbb{I}_{e}\left(\left[\sigma_{z}, .\right]\right)[V, .] e^{i s\left[L_{0}^{s e m i}, .\right]}[V, .] \mathbb{1}_{e}\left(\left[\sigma_{z}, .\right]\right) P d s .
$$

\subsection{Lindbladian of the spin-boson system}

Let

$$
\mathcal{L}=i K^{\sharp}
$$

The aim of this subsection is to give an explicit formula of $\mathcal{L}$. Moreover, we prove that this operator has the form of a Lindblad generator (or Lindbladian).

Let us introduce the well known formula of distribution theory

$$
\int_{0}^{\infty} e^{ \pm i t \omega} d t=\frac{ \pm i}{\omega \pm i 0}=\pi \delta(\omega) \pm i V_{p}\left(\frac{1}{\omega}\right)
$$

where

$$
\frac{1}{x+i 0}=\lim _{\varepsilon \rightarrow 0} \frac{1}{x+i \varepsilon},
$$




$$
\begin{aligned}
& \int f(x) \delta(x) d x=f(0), \\
& \int f(x) V_{p}\left(\frac{1}{x}\right) d x=\lim _{\varepsilon \rightarrow 0} \int_{|x| \geq \varepsilon} \frac{f(x)}{x} d x=P P \int \frac{f(x)}{x} d x, \\
& \int f(x) \frac{1}{x+i 0} d x=\lim _{\varepsilon \rightarrow 0} \int f(x) \frac{1}{x+i \varepsilon} d x,
\end{aligned}
$$

for all $f$, such that $\mathbb{R} \ni x \mapsto f(x)$ is a continuous function and provided that the integrals on the right are well defined and that the limits exist.

Note that the eigenvalues of $\left[\sigma_{z},.\right]$ are 2, -2 and 0 where 2, -2 are non degenerate and 0 is of multiplicity two. Besides, the corresponding eigenvectors are respectively given by $\left|\Psi_{+}><\Psi_{-}\right|,\left|\Psi_{-}><\Psi_{+}\right|$and $\left|\Psi_{+}><\Psi_{+}\right|,\left|\Psi_{-}><\Psi_{-}\right|$. Let us set

$$
\begin{aligned}
& n_{+}=\left(\begin{array}{ll}
1 & 0 \\
0 & 0
\end{array}\right), n_{-}=\left(\begin{array}{ll}
0 & 0 \\
0 & 1
\end{array}\right), \sigma_{+}=\left(\begin{array}{ll}
0 & 1 \\
0 & 0
\end{array}\right), \sigma_{-}=\left(\begin{array}{ll}
0 & 0 \\
1 & 0
\end{array}\right), \\
& n_{+}^{L} X=n_{+} X, n_{+}^{R} X=X n_{+}, n_{-}^{L} X=n_{-} X, n_{-}^{R} X=X n_{-}, \\
& N(\omega)=\frac{1}{e^{\beta \omega(k)}-1} .
\end{aligned}
$$

It is easy to check that

$$
\begin{aligned}
& \mathbb{I}_{2}\left(\left[\sigma_{z}, .\right]\right)=n_{+}^{L} n_{-}^{R}, \\
& \mathbb{I}_{-2}\left(\left[\sigma_{z}, .\right]\right)=n_{-}^{L} n_{+}^{R}, \\
& \mathbb{I}_{0}\left(\left[\sigma_{z}, .\right]\right)=n_{+}^{L} n_{+}^{R}+n_{-}^{L} n_{-}^{R} .
\end{aligned}
$$

The explicit formula of the Lindbladian associated to the spin-boson system is given as follows.

Theorem 3.10 If the following assumptions are met

i) $\int_{0}^{\infty}|h(t)| d t<\infty$

ii) $\alpha$ is a $\mathcal{C}^{1}$ function in a neighbourhood of the sphere $B(0,2)=\left\{k \in \mathbb{R}^{3},|k|=2\right\}$,

iii) $(1+\omega) \alpha \in L^{\infty}\left(\mathbb{R}^{3}\right)$,

then for all $X \in M_{2}$,

$$
\begin{aligned}
\mathcal{L}(X) & =i\left(\operatorname{Im}(\alpha, \alpha)_{+}^{-}-\operatorname{Im}(\alpha, \alpha)_{-}^{+}\right)\left[n_{+}, X\right] \\
& +i\left(\operatorname{Im}(\alpha, \alpha)_{-}^{-}-\operatorname{Im}(\alpha, \alpha)_{+}^{+}\right)\left[n_{-}, X\right] \\
& +\operatorname{Re}(\alpha, \alpha)_{-}^{+}\left(2 \sigma_{+} X \sigma_{-}-\left\{n_{+}, X\right\}\right) \\
& +\operatorname{Re}(\alpha, \alpha,)_{-}^{-}\left(2 \sigma_{-} X \sigma_{+}-\left\{n_{-}, X\right\}\right),
\end{aligned}
$$

where

$$
\begin{aligned}
& \operatorname{Im}(\alpha, \alpha)_{+}^{+}=\int_{\mathbb{R}^{3}} \frac{N(\omega)+1}{\omega+2}|\alpha(k)|^{2} d k, \\
& \operatorname{Im}(\alpha, \alpha)_{-}^{-}=P P \int \frac{N(\omega)}{\omega-2}|\alpha(k)|^{2} d k,
\end{aligned}
$$




$$
\begin{aligned}
& \operatorname{Im}(\alpha, \alpha)_{-}^{+}=P P \int \frac{N(\omega)+1}{\omega-2}|\alpha(k)|^{2} d k, \\
& \operatorname{Im}(\alpha, \alpha)_{+}^{-}=\int_{\mathbb{R}^{3}} \frac{N(\omega)}{\omega+2}|\alpha(k)|^{2} d k, \\
& \operatorname{Re}(\alpha, \alpha)_{-}^{+}=\pi \frac{e^{2 \beta}}{e^{2 \beta}-1} \int_{\mathbb{R}^{3}}|\alpha(k)|^{2} \delta(\omega-2) d k, \\
& \operatorname{Re}(\alpha, \alpha)_{-}^{-}=\frac{\pi}{e^{2 \beta}-1} \int_{\mathbb{R}^{3}}|\alpha(k)|^{2} \delta(\omega-2) d k .
\end{aligned}
$$

\section{Proof}

A straightforward computation shows that for all $X \in M_{2}$,

$$
\begin{aligned}
& \mathbb{I}_{2}\left(\left[\sigma_{z}, .\right]\right)[V, .] e^{i s\left[L_{0}^{s e m i}, .\right]}[V, .] \mathbb{I}_{2}\left(\left[\sigma_{z}, .\right]\right) P X= \\
& {\left[\varphi_{A W}(\alpha) \varphi_{A W}\left(e^{i s \omega} \alpha\right)+\varphi_{A W}\left(e^{i s \omega} \alpha\right) \varphi_{A W}(\alpha)\right] n_{+} X n_{-},} \\
& \mathbb{I}_{-2}\left(\left[\sigma_{z}, .\right]\right)[V, .] e^{i s\left[L_{0}^{s e m i}, .\right]}[V, .] \mathbb{I}_{-2}\left(\left[\sigma_{z}, .\right]\right) P X= \\
& {\left[\varphi_{A W}(\alpha) \varphi_{A W}\left(e^{i s \omega} \alpha\right)+\varphi_{A W}\left(e^{i s \omega} \alpha\right) \varphi_{A W}(\alpha)\right] n_{-} X n_{+},} \\
& \mathbb{I}_{0}\left(\left[\sigma_{z}, .\right]\right)[V, .] e^{i s\left[L_{0}^{s e m i}, .\right]}[V, .] \mathbb{I}_{0}\left(\left[\sigma_{z}, .\right]\right) P X= \\
& {\left[e^{-2 i s} \varphi_{A W}(\alpha) \varphi_{A W}\left(e^{i s \omega} \alpha\right)+e^{2 i s} \varphi_{A W}\left(e^{i s \omega} \alpha\right) \varphi_{A W}(\alpha)\right] n_{+} X n_{+}+} \\
& {\left[e^{2 i s} \varphi_{A W}(\alpha) \varphi_{A W}\left(e^{i s \omega} \alpha\right)+e^{-2 i s} \varphi_{A W}\left(e^{i s \omega} \alpha\right) \varphi_{A W}(\alpha)\right] n_{-} X n_{-}-} \\
& {\left[e^{-2 i s} \varphi_{A W}(\alpha) \varphi_{A W}\left(e^{i s \omega} \alpha\right)+e^{2 i s} \varphi_{A W}\left(e^{i s \omega} \alpha\right) \varphi_{A W}(\alpha)\right] \sigma_{+} X \sigma_{-}-} \\
& {\left[e^{2 i s} \varphi_{A W}(\alpha) \varphi_{A W}\left(e^{i s \omega} \alpha\right)+e^{-2 i s} \varphi_{A W}\left(e^{i s \omega} \alpha\right) \varphi_{A W}(\alpha)\right] \sigma_{-} X \sigma_{+} .}
\end{aligned}
$$

Hence for all $X \in M_{2}$, we have

$$
\begin{aligned}
& \sum_{e \in s p\left(\left[\sigma_{z}, .\right]\right)} e^{-i s e} P \mathbb{I}_{e}\left(\left[\sigma_{z}, .\right]\right)[V, .] e^{i s\left[L_{0}^{s e m i}, .\right]}[V, .] \mathbb{1}_{e}\left(\left[\sigma_{z}, .\right]\right)(X)= \\
& {\left[e^{-2 i s}<\varphi_{A W}(\alpha) \varphi_{A W}\left(e^{i s \omega} \alpha\right)>+e^{-2 i s}<\varphi_{A W}\left(e^{i s \omega} \alpha\right) \varphi_{A W}(\alpha)>\right] n_{+} X n_{-}+} \\
& {\left[e^{2 i s}<\varphi_{A W}(\alpha) \varphi_{A W}\left(e^{i s \omega} \alpha\right)>+e^{2 i s}<\varphi_{A W}\left(e^{i s \omega} \alpha\right) \varphi_{A W}(\alpha)>\right] n_{-} X n_{+}-} \\
& 2 \operatorname{Re}\left(e^{2 i s}<\varphi_{A W}\left(e^{i s \omega} \alpha\right) \varphi_{A W}(\alpha)>\right)\left[\sigma_{+} X \sigma_{-}-n_{+} X n_{+}\right]- \\
& 2 \operatorname{Re}\left(e^{-2 i s}<\varphi_{A W}\left(e^{i s \omega} \alpha\right) \varphi_{A W}(\alpha)>\right)\left[\sigma_{-} X \sigma_{+}-n_{-} X n_{-}\right] .
\end{aligned}
$$

It follows that

$$
\begin{aligned}
& \mathcal{L}(X)=-\left[\int_{0}^{\infty} e^{-2 i s}\left(<\varphi_{A W}(\alpha) \varphi_{A W}\left(e^{i s \omega} \alpha\right)>+<\varphi_{A W}\left(e^{i s \omega} \alpha\right) \varphi_{A W}(\alpha)>\right) d s\right] n_{+} X n_{-}+ \\
& {\left[-\int_{0}^{\infty} e^{2 i s}\left(<\varphi_{A W}(\alpha) \varphi_{A W}\left(e^{i s \omega} \alpha\right)>+<\varphi_{A W}\left(e^{i s \omega} \alpha\right) \varphi_{A W}(\alpha)>\right) d s\right] n_{-} X n_{+}+} \\
& 2 \operatorname{Re}\left(\int_{0}^{\infty} e^{2 i s}<\varphi_{A W}\left(e^{i s \omega} \alpha\right) \varphi_{A W}(\alpha)>d s\right)\left[\sigma_{+} X \sigma_{-}-n_{+} X n_{+}\right]+ \\
& 2 \operatorname{Re}\left(\int_{0}^{\infty} e^{-2 i s}<\varphi_{A W}\left(e^{i s \omega} \alpha\right) \varphi_{A W}(\alpha)>d s\right)\left[\sigma_{-} X \sigma_{+}-n_{-} X n_{-}\right] .
\end{aligned}
$$


But we have

$$
\begin{aligned}
<\varphi_{A W}(\alpha) \varphi_{A W}\left(e^{i s \omega} \alpha\right)> & =\int_{\mathbb{R}^{3}} e^{i s \omega}(N(\omega)+1)|\alpha(k)|^{2} d k+\int_{\mathbb{R}^{3}} e^{-i s \omega} N(\omega)|\alpha(k)|^{2} d k \\
& =\frac{<\varphi_{A W}\left(e^{i s \omega} \alpha\right) \varphi_{A W}(\alpha)>.}{}
\end{aligned}
$$

Now by assumptions i), ii) and iii) of the above theorem, we apply formula (12) to get

$$
\begin{aligned}
& \int_{0}^{\infty} e^{-2 i s}<\varphi_{A W}(\alpha) \varphi_{A W}\left(e^{i s \omega} \alpha\right)>d s=\operatorname{Re}(\alpha, \alpha)_{-}^{+}+i \operatorname{Im}(\alpha, \alpha)_{-}^{+}-i \operatorname{Im}(\alpha, \alpha)_{+}^{-}, \\
& \int_{0}^{\infty} e^{-2 i s}<\varphi_{A W}\left(e^{i s \omega} \alpha\right) \varphi_{A W}(\alpha)>d s=\operatorname{Re}(\alpha, \alpha)_{-}^{-}+i \operatorname{Im}(\alpha, \alpha,)_{-}^{-}-i \operatorname{Im}(\alpha, \alpha)_{+}^{+}, \\
& \int_{0}^{\infty} e^{2 i s}<\varphi_{A W}\left(e^{i s \omega} \alpha\right) \varphi_{A W}(\alpha)>d s=\operatorname{Re}(\alpha, \alpha)_{-}^{+}-i \operatorname{Im}(\alpha, \alpha)_{-}^{+}+i \operatorname{Im}(\alpha, \alpha)_{+}^{-}, \\
& \int_{0}^{\infty} e^{2 i s}<\varphi_{A W}(\alpha) \varphi_{A W}\left(e^{i s \omega} \alpha\right)>d s=\operatorname{Re}(\alpha, \alpha)_{-}^{-}+i \operatorname{Im}(\alpha, \alpha)_{+}^{+}-i \operatorname{Im}(\alpha, \alpha)_{-}^{-} .
\end{aligned}
$$

Therefore

$$
\begin{aligned}
& \mathcal{L}(X)=\left\{-\operatorname{Re}(\alpha, \alpha)_{-}^{+}-\operatorname{Re}(\alpha, \alpha)_{-}^{-}+i\left(\operatorname{Im}(\alpha)_{+}^{-}-\operatorname{Im}(\alpha, \alpha)_{-}^{+}\right)-\right. \\
& \left.i\left(\operatorname{Im}(\alpha, \alpha)_{-}^{-}-\operatorname{Im}\left(\alpha, \alpha_{+}^{+}\right)\right)\right\} n_{+} X n_{-}+\left\{-\operatorname{Re}(\alpha, \alpha)_{-}^{+}-\operatorname{Re}(\alpha, \alpha)_{-}^{-}\right. \\
& \left.i\left(\operatorname{Im}(\alpha, \alpha)_{+}^{-}-\operatorname{Im}(\alpha, \alpha)_{-}^{+}\right)+i\left(\operatorname{Im}(\alpha, \alpha)_{-}^{-}-\operatorname{Im}(\alpha, \alpha)_{+}^{+}\right)\right\} n_{-} X n_{+}+ \\
& 2 \operatorname{Re}(\alpha, \alpha)_{-}^{+}\left[\sigma_{+} X \sigma_{-}-n_{+} X n_{+}\right]+2 \operatorname{Re}(\alpha, \alpha)_{-}^{-}\left[\sigma_{-} X \sigma_{+}^{-} n_{-} X n_{-}\right] .
\end{aligned}
$$

Hence we get

$$
\begin{aligned}
\mathcal{L}(X) & =i\left(\operatorname{Im}(\alpha, \alpha)_{+}^{-}-\operatorname{Im}(\alpha, \alpha)_{-}^{+}\right)\left[n_{+} X n_{-}-n_{-} X n_{+}\right] \\
& +i\left(\operatorname{Im}(\alpha, \alpha)_{-}^{-}-\operatorname{Im}(\alpha, \alpha)_{+}^{+}\right)\left[n_{-} X n_{+}-n_{+} X n_{-}\right] \\
& +\operatorname{Re}(\alpha, \alpha)_{-}^{+}\left[2 \sigma_{+} X \sigma_{-}-2 n_{+} X n_{+}-\left(n_{+} X n_{-}+n_{-} X n_{+}\right)\right] \\
& +\operatorname{Re}(\alpha, \alpha)_{-}^{-}\left[2 \sigma_{-} X \sigma_{+}-2 n_{-} X n_{-}-\left(n_{+} X n_{-}+n_{-} X n_{+}\right)\right] .
\end{aligned}
$$

Moreover we have

$$
\begin{aligned}
& n_{+} X n_{-}+n_{-} X n_{+}=\left\{n_{+}, X\right\}-2 n_{+} X n_{+}=\left\{n_{-}, X\right\}-2 n_{-} X n_{-}, \\
& n_{+} X n_{-}-n_{-} X n_{+}=\left[n_{+}, X\right], \\
& n_{-} X n_{+}-n_{+} X n_{-}=\left[n_{-}, X\right] .
\end{aligned}
$$

This proves our theorem.

\section{Properties of the quantum master equation}

In this section we state some properties of the quantum master equation associated to the spin-boson system such that quantum decoherence and quantum detailed balance condition. Note that the log-Sobolev inequality with explicit computation of optimal constants are known in this context. We refer the interested reader to $[\mathrm{C}]$. 


\subsection{Quantum master equation}

Let $\rho \in M_{2}$ be a density matrix. Then the quantum master equation of the spin-boson system is given by

$$
\begin{aligned}
\frac{d \rho(t)}{d t} & =i\left(\operatorname{Im}(\alpha, \alpha)_{-}^{+}-\operatorname{Im}(\alpha, \alpha)_{+}^{-}\right)\left[n_{+}, \rho(t)\right] \\
& +i\left(\operatorname{Im}(\alpha, \alpha)_{+}^{+}-\operatorname{Im}(\alpha, \alpha)_{-}^{-}\right)\left[n_{-}, \rho(t)\right] \\
& +\operatorname{Re}(\alpha, \alpha)_{-}^{+}\left(2 \sigma_{-} \rho(t) \sigma_{+}-\left\{n_{+}, \rho(t)\right\}\right) \\
& +\operatorname{Re}(\alpha, \alpha)_{-}^{-}\left(2 \sigma_{+} \rho(t) \sigma_{-}-\left\{n_{-}, \rho(t)\right\}\right)
\end{aligned}
$$

Let us put

$$
\rho(t)=\rho_{11}(t) n_{+}+\rho_{12}(t) \sigma_{+}+\rho_{21}(t) \sigma_{-}+\rho_{22}(t) n_{-} .
$$

Therefore the above master equation is equivalent to the following system of ordinary differential equations

$$
\begin{aligned}
\frac{d}{d t} \rho_{11}(t) & =2 \operatorname{Re}(\alpha, \alpha)_{-}^{-} \rho_{22}(t)-2 \operatorname{Re}(\alpha, \alpha)_{-}^{+} \rho_{11}(t) \\
\frac{d}{d t} \rho_{12}(t) & =\left[-i\left(\operatorname{Im}(\alpha, \alpha)_{+}^{+}-\operatorname{Im}(\alpha, \alpha)_{-}^{-}\right)+i\left(\operatorname{Im}(\alpha, \alpha)_{-}^{+}-\operatorname{Im}(\alpha, \alpha)_{+}^{-}\right)-\operatorname{Re}(\alpha, \alpha)_{-}^{-}\right. \\
& \left.-\operatorname{Re}(\alpha, \alpha)_{-}^{+}\right] \rho_{12}(t) \\
\frac{d}{d t} \rho_{21}(t) & =\left[-i\left(\operatorname{Im}(\alpha, \alpha)_{-}^{+}-\operatorname{Im}\left(\alpha, \alpha_{+}^{-}\right)\right)+i\left(\operatorname{Im}(\alpha, \alpha)_{+}^{+}-\operatorname{Im}(\alpha, \alpha)_{-}^{-}\right)-\operatorname{Re}(\alpha, \alpha)_{-}^{+}\right. \\
& \left.-\operatorname{Re}(\alpha, \alpha)_{-}^{-}\right] \rho_{21}(t) \\
\frac{d}{d t} \rho_{22}(t) & =2 \operatorname{Re}(\alpha, \alpha)_{-}^{+} \rho_{11}(t)-2 \operatorname{Re}(\alpha, \alpha)_{-}^{-} \rho_{22}(t) .
\end{aligned}
$$

Hence it is straightforward to show that the thermodynamical equilibrium state $\rho_{\beta}$ of the spin system is the only solution of the above equation.

\subsection{Quantum decoherence of the spin system}

Definition 1 We say that the dynamical evolution of a quantum system describes decoherence, if there exists an orthonormal basis of $\mathcal{H}_{s}$ such that the off-diagonal elements of its time evolved density matrix in this basis vanish as $t \rightarrow \infty$.

From the system of ordinary differential equations introduced in the previous subsection, we have

$$
\begin{aligned}
\rho_{12}(t) & =\rho_{12}(0) \exp \left(-i\left(\operatorname{Im}(\alpha, \alpha)_{+}^{-}+\operatorname{Im}(\alpha, \alpha)_{+}^{+}-\operatorname{Im}(\alpha, \alpha)_{-}^{-} \operatorname{Im}(\alpha, \alpha)_{-}^{+}\right) t\right) \\
& \times \exp \left(-\left(\operatorname{Re}(\alpha, \alpha)_{-}^{-}+\operatorname{Re}(\alpha, \alpha)_{+}^{-}\right) t\right), \\
& =\rho_{12}(0) \exp \left(-i\left(\operatorname{Im}(\alpha, \alpha)_{+}^{-}+\operatorname{Im}(\alpha, \alpha)_{+}^{+}-\operatorname{Im}(\alpha, \alpha)_{-}^{-}-\operatorname{Im}(\alpha, \alpha)_{-}^{+}\right) t\right) \\
& \times \exp \left(-\pi\left(\frac{e^{2 \beta}+1}{e^{2 \beta}-1} \int_{\mathbb{R}^{3}}|\alpha(k)|^{2} \delta(\omega-2) d k\right) t\right) \\
\rho_{21}(t) & =\rho_{21}(0) \exp \left(-i\left(\operatorname{Im}(\alpha, \alpha)_{-}^{+}+\operatorname{Im}(\alpha, \alpha)_{-}^{-}-\operatorname{Im}(\alpha, \alpha)_{+}^{-}-\operatorname{Im}(\alpha, \alpha)_{+}^{+}\right) t\right) \\
& \times \exp \left(-\left(\pi \frac{e^{2 \beta}+1}{e^{2 \beta}-1} \int_{\mathbb{R}^{3}}|\alpha(k)|^{2} \delta(\omega-2) d k\right) t\right) .
\end{aligned}
$$


Therefore the spin system describes quantum decoherence if and only if

$$
\int_{\mathbb{R}^{3}}|\alpha(k)|^{2} \delta(\omega-2) d k \neq 0 .
$$

Hence the decoherence of the spin system is controlled by the cut-off function $\alpha$.

\subsection{Quantum detailed balance condition}

Definition 2 Let $\Theta$ be a generator of a quantum dynamical semigroup written as

$$
\Theta=i[H, .]+\Theta_{0},
$$

where $H$ is a self-adjoint operator. We say that $\Theta$ satisfies a quantum detailed balance condition with respect to a stationary state $\rho$ if

i) $[H, \rho]=0$,

ii) $<\Theta_{0}(A), B>_{\rho}=<A, \Theta_{0}(B)>_{\rho}$, for all $A, B \in D\left(\Theta_{0}\right)$,

$$
\text { with }<A, B>_{\rho}=\operatorname{Tr}\left(\rho A^{*} B\right) \text {. }
$$

Now we prove the following.

Theorem 4.1 The generator $\mathcal{L}$ of the quantum dynamical semigroup $T_{t}=e^{i t K^{\sharp}}$ satisfies a quantum detailed balance condition with respect to the thermodynamical equilibrium state of the spin system

$$
\rho_{\beta}=\frac{e^{-\beta \sigma_{z}}}{\operatorname{Tr}\left(e^{-\beta \sigma_{z}}\right)}
$$

Proof Note that

$$
\mathcal{L}(A)=i[H, A]+\mathcal{L}_{D}(A)
$$

with

$$
H=\left(\operatorname{Im}(\alpha, \alpha)_{+}^{-}-\operatorname{Im}(\alpha, \alpha)_{-}^{+}\right) n_{+}+\left(\operatorname{Im}(\alpha, \alpha)_{-}^{-}-\operatorname{Im}(\alpha, \alpha)_{+}^{+}\right) n_{-},
$$

and

$$
\mathcal{L}_{D}(\rho)=\operatorname{Re}(\alpha, \alpha)_{-}^{+}\left(2 \sigma_{+} \rho \sigma_{-}-\left\{n_{+}, \rho\right\}\right)+\operatorname{Re}(\alpha, \alpha)_{-}^{-}\left(2 \sigma_{-} \rho \sigma_{+}-\left\{n_{-}, \rho\right\}\right) .
$$

Therefore it is clear that $H$ is a self-adjoint operator and $\left[H, \rho_{\beta}\right]=0$. Moreover it is straightforward to show that $\mathcal{L}_{D}$ is self-adjoint for the $<,>_{\rho_{\beta}}$ scalar product. 


\section{Return to equilibrium for the spin-boson system}

\subsection{Hamiltonian case}

In this subsection we recall the results of return to equilibrium for the spin-boson system proved in $[\mathrm{JP} 2]$.

For $f \in L^{2}\left(\mathbb{R}^{3}\right)$ we define $\tilde{f}$ on $\mathbb{R} \times S^{2}$ by

$$
\tilde{f}(s, \hat{k})=\left\{\begin{array}{cc}
-|s|^{1 / 2} \bar{f}(|s| \hat{k}), & s<0, \\
s^{1 / 2} f(s \hat{k}), & s \geq 0 .
\end{array}\right.
$$

Let us put

$$
\begin{aligned}
& \mathcal{C}(\delta)=\{z \in \mathbb{C} \text { s.t } \mid \text { Imz } \mid<\delta\}, \\
& H^{2}(\delta, \eta)=\left\{f: \mathcal{C}(\delta) \longrightarrow \eta \text { s.t }\|f\|_{H^{2}(\delta, \eta)}=\sup _{|a|<\delta} \int_{-\infty}^{+\infty}\|f(x+i a)\|_{\eta}^{2} d x<\infty\right\}
\end{aligned}
$$

where $\eta$ is a Hilbert space.

Definition 3 Let $\mathcal{M}$ be a $W^{*}$-algebra, $\tau$ a dynamics on $\mathcal{M}$ and $\omega$ a faithful normal state on $\mathcal{M}$. We say that the triple $(\mathcal{M}, \tau, \omega)$ has the property of return to equilibrium if for all $A \in \mathcal{M}$ and all normal state $\mu$, we have

$$
\lim _{t \rightarrow \infty} \mu\left(\tau^{t}(A)\right)=\omega(A)
$$

Then, in the Hamiltonian approach of the spin-boson system, the following is proved in [JP2].

Theorem 5.1 Assume that the following assumptions are satisfied

(i) $\left(\omega+\omega^{-1}\right) \alpha \in L^{2}\left(\mathbb{R}^{3}\right)$,

(ii) $\int_{S^{2}}|\alpha(2 \hat{k})|^{2} d \sigma(\hat{k})>0$, where $d \sigma$ is the surface measure on $S^{2}$,

(iii) There exists $0<\delta<\frac{2 \pi}{\beta}$ such that $\tilde{\alpha} \in H^{2}\left(\delta, L^{2}\left(S^{2}\right)\right)$.

Then, for all $\beta>0$ there exists a constant $\Lambda(\beta)>0$ which depends only on the cut-off function $\alpha$ such that the spin-boson system has the property of return to equilibrium for all $0<|\lambda|<\Lambda(\beta)$.

Remark : In the above theorem the authors show that for any fixed temperature $\beta \in$ ] $0,+\infty$ [, the spectrum of the full-Liouvillean $L_{\lambda}$ associated to the spin-boson system is absolutely continuous unifomly on $\lambda \in] 0, \Lambda(\beta)$ [ and in particular for $\lambda$ very small (weak coupling). Moreover they used the theory of pertubation of KMS-states for constructing the eigenvector of $L_{\lambda}$ associated to the eigenvalue 0 . Therefore for any fixed $\left.\beta \in\right] 0,+\infty[$, the spin-boson system weakly coupled has the property of return to equilibrium. 


\subsection{Markovian case}

We shall compare the above conditions for the return to equilibrium to the one we obtain in the Markovian approach. Set $\left(T_{t}\right)_{t \geq 0}$ be a quantum dynamical semigroup on $\mathcal{B}(\eta)$ such that its generator has the form

$$
\mathcal{L}(X)=G^{*} X+X G+\sum_{k \geq 1} L_{k}^{*} X L_{k}
$$

where $G=-\frac{1}{2} \sum_{k \geq 1} L_{k}^{*} L_{k}-i H$.

Let us put

$\mathcal{A}(T)=\left\{X \in \mathcal{B}(\eta)\right.$ s.t $T_{t}(X)=X$, for all $\left.t \geq 0\right\}$,

$\mathcal{N}(T)=\left\{X \in \mathcal{B}(\eta)\right.$ s.t $\left.T_{t}\left(X^{*} X\right)=T_{t}\left(X^{*}\right) T_{t}(X), T_{t}\left(X X^{*}\right)=T_{t}(X) T_{t}\left(X^{*}\right), \forall t \geq 0\right\}$.

The following result is useful for the study of approach to equilibrium in the Markovian case.

Theorem 5.2 (Frigerio-Verri)

If $T$ has a faithful stationary state $\rho$ and $\mathcal{N}(T)=\mathcal{A}(T)$, then

$$
w^{*}-\lim _{t \rightarrow \infty} T_{t}(X)=T_{\infty}(X), \forall X \in \mathcal{B}(\eta)
$$

where $X \rightarrow T_{\infty}(X)$ is a conditional expectation. In particular the quantum dynamical semigroup $T$ has the property of return to equilibrium.

Let us state the following result which is a special case of a theorem proved in [FR2].

Theorem 5.3 Suppose that $\left(T_{t}\right)_{t}$ is a norm continuous quantum dynamical semigroup which has a faithful normal stationary state and $H$ is a self-adjoint operator which has a pure point spectrum. Then $\left(T_{t}\right)_{t}$ has the property of return to equilibrium if and only if

$$
\left\{L_{k}, L_{k}^{*}, H, k \geq 1\right\}^{\prime}=\left\{L_{k}, L_{k}^{*}, k \geq 1\right\}^{\prime} .
$$

Applying the above result, we now prove the following.

Theorem 5.4 Suppose that the following assumptions are satisfied

i) $\operatorname{Im}(\alpha, \alpha)_{ \pm}^{ \pm}$are given by real numbers,

ii) $\int_{S^{2}}|\alpha(2 k)|^{2} d k>0$.

Then the quantum dynamical semigroup of the spin-boson system at positive temperature has the property of return to equilibrium.

\section{Proof}


Set

$$
\begin{aligned}
H & =\left(\operatorname{Im}(\alpha, \alpha)_{+}^{-}-\operatorname{Im}(\alpha, \alpha)_{-}^{+}\right) n_{+}+\left(\operatorname{Im}(\alpha, \alpha)_{-}^{-}-\operatorname{Im}(\alpha, \alpha)_{+}^{+}\right) n_{-}, \\
L_{1} & =\left(2 \operatorname{Re}(\alpha, \alpha)_{-}^{+}\right)^{1 / 2} \sigma_{-}, \\
L_{2} & =\left(2 \operatorname{Re}(\alpha, \alpha)_{-}^{-}\right)^{1 / 2} \sigma_{+}, \\
G & =-\frac{1}{2} \sum_{k=1}^{2} L_{k}^{*} L_{k}-i H .
\end{aligned}
$$

Then the Lindbladian of the spin-boson system takes the form

$$
\mathcal{L}(X)=G^{*} X+X G+\sum_{k=1}^{2} L_{k}^{*} X L_{k}
$$

for all $X \in M_{2}$.

Note that the quantum dyanmical semigroup $T$ of the spin-boson system has the thermodynamical equilibrium state $\rho_{\beta}$ of the spin system as a faithful normal stationary state. Moreover $H$ is a self-adjoint bounded operator which has a pure point spectrum and it is clear that

$$
\left\{L_{k}, L_{k}^{*}, H, k=1,2\right\}^{\prime}=\left\{L_{k}, L_{k}^{*}, k=1,2\right\}^{\prime}=\mathbb{C} I .
$$

Thus from the previous theorem, the quantum dynamical semigroup of the spin-boson system has the property of return to equilibrium.

Note that compared to the Hamiltonian approach, we have in Theorem 5.4 a simplification of conditions for return to equilibrium of the spin-boson system. So in this theorem we need only that assumptions i) and ii) are satisfied. The hypothesis i) ensures that $\operatorname{Im}(\alpha, \alpha)_{ \pm}^{ \pm}$exist and are finite while if ii $)$holds, then $\operatorname{Re}(\alpha, \alpha)_{-}^{ \pm}$are not vanishing.

\subsection{Spin-boson system at zero temperature}

In the Hamiltonian case, if a quantum dynamical system which its Liouvillean $L$ has a purely absolutely continuous spectrum, except for the simple eigenvalue 0 , then this system has the property of return to equilibrium (cf [JP2]). At inverse temperature $\beta$ $(0<\beta<\infty)$, by using the perturbation theory of KMS-states (cf [DJP]), we can give an explicit formula of the eigenstate of $L$ associated to the eigenvalue 0 . But it is not the case for zero temperature $(\beta=\infty)$. On the other hand, the ground state of the spin system is not faithful and by Theorem 5.3 we cannot conclude. Let us describe the spin-boson system at zero temperature.

At zero temperature, the Hilbert space of the spin-boson system is

$$
\mathcal{H}=\mathbb{C}^{2} \otimes \Gamma_{s}\left(L^{2}\left(\mathbb{R}^{3}\right)\right) .
$$

The free Hamiltonian is defined as

$$
h_{0}=\sigma_{z} \otimes 1+1 \otimes d \Gamma(\omega),
$$


and its full Hamiltonian with interaction is the operator

$$
h_{\lambda}=h_{0}+\lambda \sigma_{x} \otimes \varphi(\alpha)
$$

where $\alpha \in L^{2}\left(\mathbb{R}^{3}\right)$ is a test function.

The zero temperature equilibrium state of the spin system is the vector state corresponding to the ground state of $\sigma_{z}$ and it has a density matrix

$$
\rho_{\infty}=\left|\Psi_{-}><\Psi_{-}\right|
$$

The weak coupling limit of the spin-boson system at zero temperature can be proved in the same way as for positive temperature. The associated Lindbladian can be deduced from the one at positive temperature by taking $\beta=\infty$ and it has the form

$$
\mathcal{L}_{\infty}(X)=-i \nu_{1}\left[n_{+}, X\right]-i \nu_{2}\left[n_{-}, X\right]+\nu_{3}\left(2 \sigma_{+} X \sigma_{-}-\left\{n_{+}, X\right\}\right),
$$

where

$$
\begin{aligned}
\nu_{1} & =\int_{\mathbb{R}^{3}} \frac{1}{\omega+2}|\alpha(k)|^{2} d k, \\
\nu_{2} & =P P \int \frac{1}{\omega-2}|\alpha(k)|^{2} d k, \\
\nu_{3} & =\pi \int_{\mathbb{R}^{3}}|\alpha(k)|^{2} \delta(\omega-2) d k .
\end{aligned}
$$

Hence for all density matrix $\rho \in M_{2}$, the associated quantum master equation is given by

$$
\frac{d \rho(t)}{d t}=i \nu_{1}\left[n_{+}, \rho(t)\right]+i \nu_{2}\left[n_{-}, \rho(t)\right]+\nu_{3}\left(2 \sigma_{-} \rho(t) \sigma_{+}-\left\{n_{+}, \rho(t)\right\}\right)=\mathcal{L}_{\infty}^{*}(\rho(t)) .
$$

Now in order to conclude the property of return to equilibrium for the quantum dynamical semigroup associated to the spin-boson system at zero temperature, we have to show it by direct computation.

Theorem 5.5 Assume that

i) $\nu_{2}$ is given by a real number,

ii) $\int_{S^{2}}|\alpha(2 k)|^{2} d k>0$,

then the spin-boson system at zero temperature has the property of return to equilibrium. Moreover we have

$$
\lim _{t \rightarrow \infty} \operatorname{Tr}\left(e^{t \mathcal{L}_{\infty}^{*}} \rho A\right)=\operatorname{Tr}\left(\rho_{\infty} A\right),
$$

for all $A \in M_{2}$ and all $\rho$ be a given density matrix. 


\section{Proof}

Let us consider the orthonormal basis of $M_{2}$ given by

$$
\left\{\left|\Psi_{+}><\Psi_{+}\right|,\left|\Psi_{+}><\Psi_{-}\right|,\left|\Psi_{-}><\Psi_{+}\right|,\left|\Psi_{-}><\Psi_{-}\right|\right\} .
$$

Then in this basis we have

$$
e^{t \mathcal{L}_{\infty}^{*}}=\left(\begin{array}{cccc}
e^{-2 t \nu_{3}} & 0 & 0 & 0 \\
0 & e^{-t \nu_{3}} e^{i t\left(\nu_{1}-\nu_{2}\right)} & 0 & 0 \\
0 & 0 & e^{-t \nu_{3}} e^{-i t\left(\nu_{1}-\nu 2\right)} & 0 \\
-e^{-2 t \nu_{3}}+1 & 0 & 0 & 1
\end{array}\right)
$$

Therefore we get

$$
\lim _{t \rightarrow \infty} e^{t \mathcal{L}_{\infty}^{*}}=\Pi_{\infty}^{*}
$$

where

$$
\Pi_{\infty}^{*}=\left(\begin{array}{cccc}
0 & 0 & 0 & 0 \\
0 & 0 & 0 & 0 \\
0 & 0 & 0 & 0 \\
1 & 0 & 0 & 1
\end{array}\right)
$$

By direct computation, this gives

$$
\Pi_{\infty}^{*}(A)=\sigma_{-} A \sigma_{+}+n_{-} A n_{-}, \quad \forall A \in M_{2} .
$$

Now consider a density matrix $\rho$ of the form

$$
\rho=\left(\begin{array}{cc}
\alpha & \beta \\
\bar{\beta} & 1-\alpha
\end{array}\right)
$$

with $\alpha \in[0,1], \beta \in \mathbb{C}$. We have

$$
\Pi_{\infty}^{*}(\rho)=\left(\begin{array}{cc}
0 & 0 \\
0 & 1
\end{array}\right)=\left|\Psi_{-}><\Psi_{-}\right|=\rho_{\infty} .
$$

Therefore it follows that

$$
\lim _{t \rightarrow \infty} \operatorname{Tr}\left(e^{t \mathcal{L}_{\infty}^{*}} \rho A\right)=\operatorname{Tr}\left(\Pi_{\infty}^{*}(\rho) A\right)=\operatorname{Tr}\left(\rho_{\infty} A\right),
$$

$\forall A \in M_{2}$. This proves our theorem.

\section{Quantum Langevin equation and associated Hamil- tonian}

It is shown in [HP] that any quantum master equation of a simple quantum system $\mathcal{H}_{S}$ can be dilated into a unitary quantum Langevin equation (quantum stochastic differential equation) on a larger space $\mathcal{H}_{S} \otimes \Gamma$ where $\Gamma$ is a Fock space in which are naturally living quantum noises. Note that in the literature it is shown that natural quantum stochastic differential equations can be obtained from the stochastic limit of the full Hamiltonian system which is developped in [ALV].

Now let us introduce some notations which we will need in the sequel. 


\subsection{Basic notation}

Let $\mathcal{Z}$ be a Hilbert space for which we fix an orthonormal basis $\left\{z_{k}, k \in J\right\}$. We denote by $\Gamma_{s}\left(\mathbb{R}_{+}\right)$, the symmetric Fock space constructed over the Hilbert space $\mathcal{Z} \otimes L^{2}\left(\mathbb{R}_{+}\right)$. Therefore from the following identification

$$
\mathcal{Z} \otimes L^{2}\left(\mathbb{R}_{+}\right) \simeq L^{2}\left(\mathbb{R}_{+}, \mathcal{Z}\right) \simeq L^{2}\left(\mathbb{R}_{+} \times J\right),
$$

we get

$$
\Gamma_{s}\left(\mathbb{R}_{+}\right)=\Gamma_{\operatorname{sym}}\left(L^{2}\left(\mathbb{R}_{+} \times J\right)\right) .
$$

The space $\mathcal{Z}$ is called the multiplicity space and $\operatorname{dim} \mathcal{Z}$ is called the multiplicity. The set $J$ is equal to $\{1, \ldots, N\}$ in the case of finite multiplicity $N$ and is equal to $\mathbb{N}$ in the case of infinite multiplicity.

Now we introduce another Hilbert space $\mathcal{H}$ called initial or system space and we identify the tensor product

$$
\mathcal{K}\left(\mathbb{R}_{+}\right)=\mathcal{H} \otimes \Gamma_{s}\left(\mathbb{R}_{+}\right)=\mathcal{H} \otimes \bigoplus_{n=0}^{\infty} L^{2}\left(\mathbb{R}_{+} \times J\right)^{\otimes n}=\bigoplus_{n=0}^{\infty} \mathcal{H} \otimes L^{2}\left(\mathbb{R}_{+} \times J\right)^{\otimes n}
$$

with the direct sum

$$
\bigoplus_{n=0}^{\infty} \mathcal{H} \otimes L_{\mathrm{Sym}}^{2}\left(\left(\mathbb{R}_{+} \times J\right)^{n}\right) \simeq \bigoplus_{n=0}^{\infty} L_{\mathrm{sym}}^{2}\left(\left(\mathbb{R}_{+} \times J\right)^{n}, \mathcal{H}\right)
$$

consisting of vector $\Psi=\left(\Psi_{n}\right)_{n \geq 0}$ such that $\Psi_{n} \in L_{\text {Sym }}^{2}\left(\left(\mathbb{R}_{+} \times J\right)^{n}, \mathcal{H}\right)$ and

$$
\|\Psi\|_{\mathcal{K}\left(\mathbb{R}_{+}\right)}^{2}=\sum_{n \geq 0} \frac{1}{n !}\left\|\Psi_{n}\right\|_{L_{\text {Sym }}^{2}\left(\left(\mathbb{R}_{+} \times J\right)^{n}, \mathcal{H}\right)}^{2}<\infty .
$$

Note that for $f \in L^{2}\left(\mathbb{R}_{+} \times J\right)$, we define its associated exponential vector by

$$
\varepsilon(f)=\sum_{n \geq 0} \frac{f^{\otimes n}}{\sqrt{n !}} .
$$

\subsection{Hudson-Parthasarathy equation}

Let $H, R_{k}$ and $S_{k l}, k, l \geq 1$ be bounded operators on $\mathcal{H}$ such that

$$
H=H^{*}, \sum_{j} S_{j k}^{*} S_{j l}=\sum_{j} S_{k j} S_{l j}^{*}=\delta_{k l},
$$

and the sum $\sum_{k} R_{k}^{*} R_{k}$ are assumed to be strongly convergent to a bounded operator. Through $H, R_{k}$ and $S_{k l}$ we define the following operators

$$
S \in \mathcal{U}(\mathcal{H} \otimes \mathcal{Z}), R \in \mathcal{B}(\mathcal{H}, \mathcal{H} \otimes \mathcal{Z}), G \in \mathcal{B}(\mathcal{H})
$$

by 


$$
\begin{aligned}
& R u=\sum_{k}\left(R_{k} u\right) \otimes z_{k}, \forall u \in \mathcal{H}, \\
& S=\sum_{k l} S_{k l} \otimes\left|z_{k}><z_{l}\right|, \\
& G=-i H-\frac{1}{2} \sum_{k} R_{k}^{*} R_{k}=-i H-\frac{1}{2} R^{*} R .
\end{aligned}
$$

The basic quantum noises are the processes

$$
\begin{aligned}
A_{i}(t) & =A\left(\mathbb{I}_{(0, t)} \otimes z_{i}\right) \\
A_{i}^{+}(t) & =A^{+}\left(\mathbb{1}_{(0, t)} \otimes z_{i}\right), \\
\Lambda_{i j}(t) & =\Lambda\left(\pi_{(0, t)} \otimes\left|z_{i}><z_{j}\right|\right),
\end{aligned}
$$

where $i, j \in J, \mathbb{I}_{(0, t)}$ is the indicator function over $(0, t)$, while $\pi_{(0, t)}$ is the multipication operator by $\mathbb{I}_{(0, t)}$ in $L^{2}\left(\mathbb{R}_{+}\right)$.

The Hudson-Parthasarathy equation is defined as follows

$(\mathrm{H}-\mathrm{P})\left\{\begin{array}{l}d U(t)=\left\{\sum_{k} R_{k} d A_{k}^{+}(t)+\sum_{k l}\left(S_{k l}-\delta_{k l}\right) d \Lambda_{k l}(t)-\sum_{k l} R_{k}^{*} S_{k l} d A_{l}(t)+G d t\right\} U(t) \\ U(0)=1\end{array}\right.$

Note that in order to have a unitary solution $U$ of (H-P), we need some conditions on the system operators. Actually the following theorem holds.

Theorem 6.1 Suppose that the system operators $H, R_{k}, S_{k l}$ satisfies (16). Then there exists a unique strongly continuous unitary adapted process $U(t)$ which satisfies equation $(H-P)$.

\section{Proof}

For the proof of this theorem we refer the reader to $[\mathrm{P}]$.

Now in order to associate a group $V$ to the solution $U$ of $(\mathrm{H}-\mathrm{P})$, we first introduce the one-parameter strongly continuous unitary group $\theta$ in $L^{2}(\mathbb{R}, \mathcal{Z})$ and its associated second quantization $\Theta$ in $\Gamma(\mathbb{R})$, defined by

$$
\begin{aligned}
\theta_{t} f(r) & =f(r+t), \quad \forall f \in L^{2}(\mathbb{R}, \mathcal{Z}), \\
\Theta_{t} e(f) & =e\left(\theta_{t} f\right), \quad \forall f \in L^{2}(\mathbb{R}, \mathcal{Z}) .
\end{aligned}
$$

Note that $\Theta$ and $U(t)$ can be extended to act on the space

$$
\mathcal{K}(\mathbb{R})=\mathcal{H} \otimes \Gamma_{s}\left(\mathbb{R}_{+}\right) \otimes \Gamma_{s}\left(\mathbb{R}_{-}\right)=\mathcal{K}\left(\mathbb{R}_{+}\right) \otimes \Gamma_{s}\left(\mathbb{R}_{-}\right)=\mathcal{H} \otimes \Gamma_{s}(\mathbb{R}),
$$

by

$$
\begin{aligned}
& \Theta_{t}=1 \otimes \Theta_{t} \quad \text { in } \mathcal{H} \otimes \Gamma_{s}(\mathbb{R}), \\
& U(t)=U(t) \otimes 1 \quad \text { in } \mathcal{K}\left(\mathbb{R}_{+}\right) \otimes \Gamma_{s}\left(\mathbb{R}_{-}\right) .
\end{aligned}
$$

Theorem 6.2 Let $\Theta$ be the one-parameter strongly continuous group defined by (17) and $U$ the solution of the EDSQ (H-P) whith system operators satisfying (16). Then

$$
U(t+s)=\Theta_{s}^{*} U(t) \Theta_{s} U(s), \forall s, t \geq 0,
$$


and the family $V=\left\{V_{t}\right\}_{t \in \mathbb{R}}$ such that

$$
V_{t}=\left\{\begin{array}{cc}
\Theta_{t} U(t), & t \geq 0 \\
U^{*}(|t|) \Theta_{t}, & t \leq 0
\end{array}\right.
$$

defines a one-parameter strongly continuous unitary group. Furthermore, the family of two-parameter unitary operators

$$
U(t, s)=\Theta_{t}^{*} V_{t-s} \Theta_{s}=\Theta_{s}^{*} U(t-s) \Theta_{s}, \quad \forall s \leq t,
$$

is strongly continuous in $t$ and in $s$ and satisfies the composition low

$$
U(t, s) U(t, r)=U(t, r), \quad \forall r \leq s \leq t .
$$

\section{Proof}

See $[B]$ for the proof of this theorem.

The group $V$ defined as above, describes the reversible evolution of the small system plus the reservoir which is modelized by the free bose gas. The free evolution of the reservoir is represented by the group $\Theta$ whose generator is formally given by

$$
E_{0}=d \Gamma\left(i \frac{\partial}{\partial x}\right)
$$

Note that $U(t)=U(t, 0)=\Theta_{t}^{*} V_{t}$ is the evolution operator giving the dynamics state from time 0 to time $t$ of the whole system in the interaction picture. Moreover by the Stone theorem

$$
\begin{aligned}
d \Theta_{t} & =-i E_{0} \Theta_{t} d t \\
d V_{t} & =-i K V_{t} d t .
\end{aligned}
$$

The operators $H, E_{0}$ represent respectively the associated energy to the small system and the reservoir. While the operator $K$ represent the total energy of the combined system in the interaction picture and the system operators $R_{j}, S_{i j}$ control this interaction. Besides, if we take $R_{j}=0, S_{i j}=\delta_{i j}$, then we get

$$
U(t)=e^{i t H}, V_{t}=e^{-i t E_{0}} e^{-i t H},
$$

and $K=E_{0}+H$ which is self-adjoint operator defined on $\mathcal{H} \otimes D\left(E_{0}\right)$.

In [G], Gregoratti give a essentially self-adjoint restriction of the Hamiltonian $K$ which appears as a singular perturbation of $E_{0}+H$.

\subsection{Hamiltonian associated to the Hudson-Parthasarathy equa- tion}

Let us recall that the generators $\epsilon_{0}$ and $E_{0}$ of the groups $\theta$ in $L^{2}(\mathbb{R}, \mathcal{Z})$ and $\Theta$ in $\mathcal{K}$ are self-adjoint unbounded operators. In order to explicit their domains we introduce the Sobolev space

$$
H^{\sum}\left((\mathbb{R} \times J)^{n}, \mathcal{H}\right)=\left\{u \in L^{2}\left((\mathbb{R} \times J)^{n}, \mathcal{H}\right) \text { such that } \sum_{k=1}^{n} \partial_{k} u \in L^{2}\left((\mathbb{R} \times J)^{n}, \mathcal{H}\right)\right\}
$$


where all the derivatives of $u$ are in the sense of distributions in $(\mathbb{R} \times J)^{n}(n \geq 1)$ and

$$
H^{\sum}\left((\mathbb{R} \times J)^{0}, \mathcal{H}\right)=\mathcal{H}
$$

Furthermore $H^{\sum}\left((\mathbb{R} \times J)^{n}, \mathcal{H}\right)$ is a Hilbert space with respect to the scalar product

$$
<u, v>_{H \Sigma\left((\mathbb{R} \times J)^{n}, \mathcal{H}\right)}=<u, v>_{L^{2}\left((\mathbb{R} \times J)^{n}, \mathcal{H}\right)}+<\sum_{k=1}^{n} \partial_{k} u, \sum_{k=1}^{n} \partial_{k} v>_{L^{2}\left((\mathbb{R} \times J)^{n}, \mathcal{H}\right)} .
$$

Set

$$
H_{\mathrm{sym}}^{\sum}\left((\mathbb{R} \times J)^{n}, \mathcal{H}\right)=H^{\sum}\left((\mathbb{R} \times J)^{n}, \mathcal{H}\right) \cap L_{\text {sym }}^{2}\left((\mathbb{R} \times J)^{n}, \mathcal{H}\right)
$$

We have

$$
D\left(\epsilon_{0}\right)=H^{1}(\mathbb{R}, \mathcal{Z}), \text { and } \epsilon_{0} u=i u^{\prime},
$$

While the domain of $E_{0}$ is given by

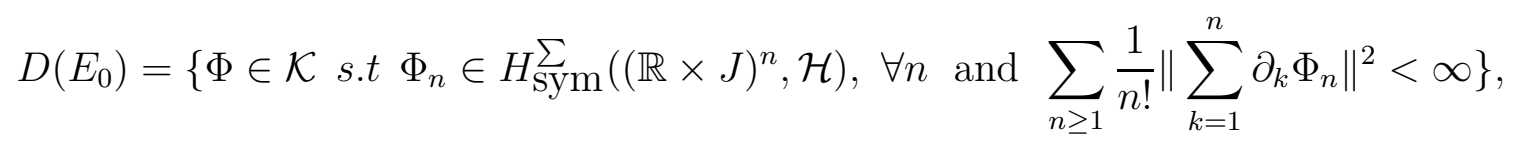

and this operator acts on its domain by $\left(E_{0} \Phi\right)_{n}=i \sum_{k=1}^{n} \partial_{k} \Phi_{n}$.

Set $\mathbb{R}_{*}=\mathbb{R} \backslash\{0\}$. Let us introduce the following dense subspaces in $\mathcal{K}$ defined as

$$
\begin{aligned}
\mathcal{W} & =\left\{\Phi \in \mathcal{K} \text { s.t } \Phi_{n} \in H_{\text {sym }}\left(\left(\mathbb{R}_{*} \times J\right)^{n}, \mathcal{H}\right), \forall n \text { and } \sum_{n \geq 1} \frac{1}{n !}\left\|\sum_{k=1}^{\infty} \partial_{k} \Phi_{n}\right\|_{L^{2}(\mathbb{R} \times J)^{n}, \mathcal{H}}^{2}<\infty\right\}, \\
\nu_{s} & =\left\{\Phi \in W \text { s.t } \sum_{n \geq 0} \frac{1}{n !}\left\|\left.\Phi_{n+1}\right|_{\left\{r_{n+1}=s\right\}}\right\|_{\mathcal{Z} \otimes L^{2}\left((\mathbb{R} \times J)^{n}, \mathcal{H}\right)}^{2}<\infty\right\}, \\
\nu_{0^{ \pm}} & =\nu_{0^{-}} \cap \nu_{0^{+}},
\end{aligned}
$$

where $\left.\Phi_{n+1}\right|_{\left\{r_{n+1}=s\right\}}$ is the trace (restriction) of the function $\Phi_{n+1}$ on the hyperplane $\left\{r_{n+1}=s\right\}$, for all $s \in \mathbb{R}_{*} \cup\left\{0^{-}, 0^{+}\right\}$. Clearly

$$
\nu_{0^{ \pm}} \subseteq \mathcal{W} .
$$

Now we define the trace operator $a(s): \nu_{s} \longrightarrow \mathcal{Z} \otimes \mathcal{K}$ such that

$$
(a(s) \Phi)_{n}=\left.\Phi_{n+1}\right|_{\left\{r_{n+1}=s\right\}} .
$$

Note that $\varepsilon\left(H^{1}\left(\mathbb{R}^{*}, \mathcal{Z}\right)\right) \subset \nu_{s}$ and

$$
a(s) \Psi(u) \otimes h=u(s) \otimes \Psi(u) \otimes h, \quad \forall u \in H^{1}\left(\mathbb{R}_{*}, \mathcal{Z}\right), h \in \mathcal{H},
$$

where

$$
\Psi(u)=\left(1, u, u^{\otimes 2}, \ldots, u^{\otimes n}, \ldots\right) .
$$

Moreover $\mathcal{W} \supset D\left(E_{0}\right)$ and $E_{0}$ can be extended to a non-symmetric unbounded operator in $\mathcal{W}$ by

$$
(E \Phi)_{n}=i \sum_{k=1}^{n} \partial_{k} \Phi_{n}
$$

The following theorem gives an essentially self-adjoint restriction of the Hamiltonian operator associated to (H-P) and it is proved in $[\mathrm{G}]$. 
Theorem 6.3 Let $K$ be the Hamiltonian operator associated to the equation (H-P) such that the system operators satisfying (16). Then

(1) $D(K) \cap \nu_{0^{ \pm}}=\left\{\Phi \in \nu_{0^{ \pm}}\right.$s.t $\left.a\left(0^{-}\right) \Phi=S a\left(0^{+}\right) \Phi+R \Phi\right\}$,

(2) $K \Phi=\left(H+E-i R^{*} a\left(0^{-}\right)+\frac{i}{2} R^{*} R\right) \Phi, \quad \forall \Phi \in D(K) \cap \nu_{0^{ \pm}}$,

(3) $\left.K\right|_{D(K) \cap \nu_{0 \pm}}$ is a essentially sef-adjoint operator.

\subsection{Hamiltonian associated to the stochastic evolution of the spin-boson system}

Recall that the quantum Langevin equation of the spin-boson system is defined on $\mathbb{C}^{2} \otimes \Gamma_{s}\left(L^{2}\left(\mathbb{R}_{+}, \mathbb{C}^{2}\right)\right)$ by

$$
\left\{\begin{array}{l}
d U(t)=\left\{G d t+\sum_{k=1}^{2} L_{k} d A_{k}^{+}(t)-\sum_{k=1}^{2} L_{k}^{*} d A_{k}(t)\right\} U(t) \\
U(0)=I
\end{array}\right.
$$

where $G, L_{k}, k \in\{0,1\}$ are given by the relation (14).

Note that this equation satisfies the class of Hudson-Parthasarathy equation with $S_{i j}=$ $\delta_{i j}$. Moreover we have

$$
\begin{aligned}
& S=I \\
& R u=\left(2 \operatorname{Re}(\alpha, \alpha)_{-}^{+}\right)^{1 / 2} \sigma_{-} u \otimes \Psi_{+}+\left(2 \operatorname{Re}(\alpha, \alpha)_{-}^{-}\right)^{1 / 2} \sigma_{+} u \otimes \Psi_{-}, \forall u \in \mathbb{C}^{2}, \\
& R^{*} u \otimes \varphi=<\Psi_{+}, \varphi>\left(2 \operatorname{Re}(\alpha, \alpha)_{-}^{+}\right)^{1 / 2} \sigma_{+} u+<\Psi_{-}, \varphi>\left(2 \operatorname{Re}(\alpha, \alpha)_{-}^{-}\right)^{1 / 2} \sigma_{-} u, \\
& \forall u, \varphi \in \mathbb{C}^{2}, \\
& R^{*} R=2 \operatorname{Re}(\alpha, \alpha)_{-}^{+} n_{+}+2 \operatorname{Re}(\alpha, \alpha)_{-}^{-} n_{-} .
\end{aligned}
$$

Therefore we get

$$
\nu_{0^{ \pm}} \cap D(K)=\left\{\Phi \in \nu_{0^{ \pm}} \quad \text { s.t } a\left(0^{-}\right) \Phi=a\left(0^{+}\right) \Phi+R \Phi\right\}
$$

and

$$
K \Phi=\left(H+E-i R^{*} a\left(0^{-}\right)+i\left(\operatorname{Re}(\alpha, \alpha)_{-}^{+} n_{+}+\operatorname{Re}(\alpha, \alpha)_{-}^{-} n_{-}\right)\right) \Phi, \quad \forall \Phi \in \nu_{0^{ \pm}} \cap D(K) .
$$

Let us recall that the associated energy of the reservoir is given by $E=d \Gamma\left(i \frac{\partial}{\partial x}\right)$. Therefore by using spectral theorem, we have $i \frac{\partial}{\partial x}$ is a multiplication operator by a variable $\omega$ in $\mathbb{R}$. Thus we get

$$
E=d \Gamma(\omega),
$$

and $E$ is the same as the usual Hamiltonian. On the other hand, the operator

$$
H=\left(r m \operatorname{Im}(\alpha / \alpha)_{+}^{-}-\operatorname{Im}(\alpha / \alpha)_{-}^{+}\right) n_{+}+\left(\operatorname{Im}(\alpha / \alpha)_{-}^{-}-\operatorname{Im}(\alpha / \alpha)_{+}^{+}\right),
$$

describes the energy of the spin. Note that the constants $\operatorname{Im}(\alpha / \alpha)_{ \pm}^{ \pm}$have an important physical interpretation. In some sense they contain all the physical informations on the original Hamiltonian of the spin. The free evolution of the combined system is described by $\mathcal{H}_{f}=H+E$ and the Hamiltonian $K$ appears as a singular perturbation of $H_{f}$, where the operator $R$ defined as above control the interaction between the spin and the reservoir. 


\section{$7 \quad$ Repeated quantum interaction model}

In this section, we start by describing the repeated quantum interaction model (cf $[\mathrm{AP}]$ ). We prove that the quantum Langevin equation of the spin-boson system at zero temperature can be obtained as the continuous limit of an Hamiltonian repeated interaction model. Moreover we compare the Lindbladian of the spin-boson system at positive temperature to the one obtained by using the method introduced in [AJ].

Let us consider a small system $\mathcal{H}_{0}$ coupled with a piece of environment $\mathcal{H}$. The interaction between the two systems is described by the Hamiltonian $H$ which is defined on $\mathcal{H}_{0} \otimes \mathcal{H}$. The associated unitary evolution during the interval $[0, h]$ of times is

$$
\mathbb{L}=e^{-i h H} .
$$

After the first interaction, we repeat this time coupling the same $\mathcal{H}_{0}$ with a new copy of $\mathcal{H}$. Therefore, the sequence of the repeated interactions is described by the space

$$
\mathcal{H}_{0} \otimes \bigotimes_{\mathbb{N}^{*}} \mathcal{H}
$$

The unitary evolution of the small system in interaction picture with the $n-t h$ copy of $\mathcal{H}$, denoted by $\mathcal{H}_{n}$, is the operator $\mathbb{L}_{n}$ which acts as $\mathbb{L}$ on $\mathcal{H}_{0} \otimes \mathcal{H}_{n}$ and acts as the identity on copy of $\mathcal{H}$ other than $\mathcal{H}_{n}$. The associated evolution equations of this model is defined on $\mathcal{H}_{0} \otimes \bigotimes_{\mathbb{N}^{*}} \mathcal{H}$ by

$$
\left\{\begin{array}{l}
u_{n+1}=\mathbb{L}_{n+1} u_{n} \\
u_{0}=I
\end{array}\right.
$$

Let $\left\{X_{i}\right\}_{1 \in \Lambda \cup\{0\}}$ be an orthonormal basis of $\mathcal{H}$ with $X_{0}=\Omega$ and let us consider the coefficients $\left(\mathbb{L}_{j}^{i}\right)_{i, j \in \Lambda \cup\{0\}}$ which are operators on $\mathcal{H}_{0}$ of the matrix representation of $\mathbb{L}$ in the basis $\left\{X_{i}\right\}_{i \in \Lambda \cup\{0\}}$.

\section{Theorem 7.1 If}

$$
\begin{aligned}
\mathbb{L}_{0}^{0} & =I-h\left(i H+\frac{1}{2} \sum_{k} L_{k}^{*} L_{k}\right)+h \omega_{0}^{0}, \\
\mathbb{L}_{j}^{0} & =\sqrt{h} L_{j}+\sqrt{h} \omega_{j}^{0}, \\
\mathbb{L}_{0}^{i} & =-\sqrt{h} \sum_{k} L_{k}^{*} S_{i}^{k}+\sqrt{h} \omega_{0}^{i}, \\
\mathbb{L}_{j}^{i} & =S_{j}^{i}+h \omega_{j}^{i} .
\end{aligned}
$$

where $H$ is a self-adjoint bounded operator, $\left(S_{j}^{i}\right)_{i, j}$ is a family of unitary operator, $\left(L_{i}\right)_{i}$ are operators on $\mathcal{H}_{0}$ and the terms $\omega_{j}^{i}$ converge to 0 when $h$ tends to 0 , then the solution $\left(u_{n}\right)_{n \in \mathbb{N}}$ of (19) is made of invertible operators which are locally uniformely bounded in norm. Moreover $u_{[t / h]}$ converges weakly to the solution $U(t)$ of the equation

$$
\left\{\begin{array}{l}
d U(t)=\sum_{i, j} L_{j}^{i} U(t) d a_{j}^{i}(t) \\
U(0)=I
\end{array}\right.
$$


where

$$
\begin{aligned}
L_{0}^{0} & =-\left(i H+\frac{1}{2} \sum_{k} L_{k}^{*} L_{k}\right), \\
L_{j}^{0} & =L_{j}, \\
L_{0}^{i} & =-\sum_{k} L_{k}^{*} S_{i}^{k}, \\
L_{j}^{i} & =S_{j}^{i}-\delta_{i j} I .
\end{aligned}
$$

\section{Proof}

See $[\mathrm{AP}]$ for the proof of this theorem.

Now let us put $\mathcal{H}_{0}=\mathcal{H}=\mathbb{C}^{2}$ and consider the dipole interaction Hamiltonian defined on $\mathbb{C}^{2} \otimes \mathbb{C}^{2}$ as

$$
H=\sigma_{z} \otimes I+I \otimes H_{R}+\frac{1}{\sqrt{h}}\left(\sigma_{-} \otimes a^{*}+\sigma_{+} \otimes a\right),
$$

where

$$
\begin{aligned}
H_{R} & =\left(\begin{array}{ll}
0 & 0 \\
0 & 1
\end{array}\right), \text { is the Hamiltonian of the piece of the reservoir, } \\
V & =\sigma_{-}, \\
a & =\left(\begin{array}{ll}
0 & 1 \\
0 & 0
\end{array}\right) \text { and } a^{*} \text { is the adjoint of } a .
\end{aligned}
$$

Let us fix an orthonormal basis $\{\Omega, X\}$ of $\mathbb{C}^{2}$ such that

$$
\Omega=\left(\begin{array}{l}
1 \\
0
\end{array}\right), X=\left(\begin{array}{l}
0 \\
1
\end{array}\right) \text {. }
$$

The unitary evolution during the interval $[0, h]$ of time is $\mathbb{L}=e^{-i h H}$ such that

$$
\begin{aligned}
& \mathbb{L}_{0}^{0}=<\Omega, \mathbb{L} \Omega>=I-i h \sigma_{z}-\frac{1}{2} h \sigma_{+} \sigma_{-}+o(h), \\
& \mathbb{L}_{0}^{1}=<\Omega, \mathbb{L} X>=-i \sqrt{h} \sigma_{+}+o(\sqrt{h}), \\
& \mathbb{L}_{1}^{0}=<X, \mathbb{L} \Omega>=-i \sqrt{h} \sigma_{-}+o(\sqrt{h}), \\
& \mathbb{L}_{1}^{1}=<X, \mathbb{L} X>=I-i h \sigma_{z}-i h I-\frac{1}{2} h \sigma_{-} \sigma_{+}+o(h),
\end{aligned}
$$

Therefore we have

$$
\begin{aligned}
& \frac{\mathbb{L}_{0}^{0}-I}{h} \stackrel{h \rightarrow 0}{\longrightarrow} G_{0}=-i \sigma_{z}-\frac{1}{2} \sigma_{+} \sigma_{-}, \\
& \frac{\mathbb{L}_{0}^{1}}{\sqrt{h}} \stackrel{h \rightarrow 0}{\longrightarrow}-L^{*}=-i \sigma_{+}, \\
& \frac{\mathbb{L}_{1}^{0}}{\sqrt{h}} \stackrel{h \rightarrow 0}{\longrightarrow} L=-i \sigma_{-},
\end{aligned}
$$


Thus by Theorem 7.1 the solution $\left(u_{n}\right)_{n \in \mathbb{N}}$ of the equation

$$
\left\{\begin{array}{l}
u_{n+1}=\mathbb{L}_{n+1} u_{n} \\
u_{0}=I
\end{array}\right.
$$

is made of invertible operators which are locally uniformely bounded in norm and in particular $u_{[t / h]}$ converges weakly to the solution $U(t)$ of the equation

$$
\left\{\begin{array}{l}
d U(t)=\left\{G_{0} d t+L d A^{+}(t)-L^{*} d A^{-}(t)\right\} U(t) \\
U(0)=I
\end{array}\right.
$$

Theorem 7.2 The quantum dynamical semigroup of the repeated quantum interaction model associated to the spin-boson system at zero temperature converges towards to equilibrium.

Proof The associated Lindbladian of the above equation is of the form

$$
\mathcal{L}(X)=i\left[\sigma_{z}, X\right]+2 \sigma_{+} X \sigma_{-}-\left\{n_{+}, X\right\}
$$

and the proof is similar as the one of Theorem 5.5.

Now at inverse temperature $\beta$, we suppose that the piece of the reservoir is described by the state

$$
\rho=\frac{1}{1+e^{-\beta}} e^{-\beta H_{R}}=\left(\begin{array}{cc}
\beta_{0} & 0 \\
0 & \beta_{1}
\end{array}\right)
$$

The GNS representation of $\left(\mathbb{C}^{2}, \rho\right)$ is the triple $\left(\pi, \widetilde{\mathcal{H}}, \Omega_{R}\right)$, such that

- $\Omega_{R}=I$,

- $\widetilde{\mathcal{H}}=M_{2}$, the algebra of all complex $2 \times 2$ matrix which equipped by the scalar product

$$
<A, B>=\operatorname{Tr}\left(\rho A^{*} B\right) \text {, }
$$

- $\pi: M_{2} \longrightarrow \mathcal{B}(\widetilde{\mathcal{H}})$, such that $\pi(M) A=M A, \forall M, A \in M_{2}$.

Set

$$
X_{1}=\frac{1}{\sqrt{\beta_{1}}}\left(\begin{array}{ll}
0 & 1 \\
0 & 0
\end{array}\right), X_{2}=\frac{1}{\sqrt{\beta_{0}}}\left(\begin{array}{ll}
0 & 0 \\
1 & 0
\end{array}\right), X_{3}=\frac{1}{\sqrt{\beta_{0} \beta_{1}}}\left(\begin{array}{cc}
\beta_{1} & 0 \\
0 & -\beta_{0}
\end{array}\right) .
$$

It is easy to show that $\left(\Omega_{R}, X_{1}, X_{2}, X_{3}\right)$ is an orthonormal basis of $M_{2}$. Now, if we put $\widetilde{\mathbb{L}}=\pi(\mathbb{L})$ which is defined on $\mathbb{C}^{2} \otimes M_{2}$, then a straightforward computation shows that the coefficients $\left(\widetilde{\mathbb{L}}_{j}^{i}\right)_{i, j}$, which are operators on $\mathbb{C}^{2}$, of the matrix representation of $\widetilde{\mathbb{L}}$, are given by

$$
\begin{aligned}
& \widetilde{\mathbb{L}}_{0}^{0}=I-i h \sigma_{z}-i h \beta_{1} I-\frac{1}{2} h \beta_{0} \sigma_{+} \sigma_{-}-\frac{1}{2} h \beta_{1} \sigma_{-} \sigma_{+}+o\left(h^{2}\right), \\
& \widetilde{\mathbb{L}}_{1}^{0}=-i \sqrt{\beta_{1}} \sqrt{h} \sigma_{+}+o\left(h^{3 / 2}\right), \\
& \widetilde{\mathbb{L}}_{2}^{0}=-i \sqrt{\beta_{0}} \sqrt{h} \sigma_{-}+o\left(h^{3 / 2}\right),
\end{aligned}
$$




$$
\begin{aligned}
& \widetilde{\mathbb{L}}_{3}^{0}=o(h), \\
& \widetilde{\mathbb{L}}_{0}^{1}=-i \sqrt{\beta_{1}} \sqrt{h} \sigma_{-}+o\left(h^{3 / 2}\right), \\
& \widetilde{\mathbb{L}}_{0}^{2}=-i \sqrt{\beta_{0}} \sqrt{h} \sigma_{+}+o\left(h^{3 / 2}\right), \\
& \widetilde{\mathbb{L}}_{0}^{3}=o(h), \\
& \widetilde{\mathbb{L}}_{1}^{1}=I+o(h), \\
& \widetilde{\mathbb{L}}_{2}^{2}=I+o(h), \\
& \widetilde{\mathbb{L}}_{3}^{3}=I+o(h), \\
& \widetilde{\mathbb{L}}_{1}^{2}=\widetilde{\mathbb{L}}_{2}^{1}=\widetilde{\mathbb{L}}_{1}^{3}=\widetilde{\mathbb{L}}_{3}^{1}=\widetilde{\mathbb{L}}_{2}^{3}=\widetilde{\mathbb{L}}_{3}^{2}=0 .
\end{aligned}
$$

Therefore we get

$$
\begin{aligned}
& \frac{\widetilde{\mathbb{\mathbb { L }}}_{0}^{0}-I}{h} \stackrel{h \rightarrow 0}{\longrightarrow} L_{0}^{0}=-i \sigma_{z}-i \beta_{1} I-\frac{1}{2} \beta_{0} \sigma_{+} \sigma_{-}-\frac{1}{2} \beta_{1} \sigma_{-} \sigma_{+}, \\
& \frac{\widetilde{\mathbb{L}}_{1}^{0}}{\sqrt{h}} \stackrel{h \rightarrow 0}{\longrightarrow} L_{1}^{0}=-i \sqrt{\beta_{1}} \sigma_{+}, \\
& \frac{\widetilde{\mathbb{L}}_{2}^{0}}{\sqrt{h}} \stackrel{h \rightarrow 0}{\longrightarrow} L_{2}^{0}=-i \sqrt{\beta_{0}} \sigma_{-}, \\
& \frac{\widetilde{\mathbb{L}}_{0}^{1}}{\sqrt{h}} \stackrel{h \rightarrow 0}{\longrightarrow} L_{0}^{1}=-i \sqrt{\beta_{1}} \sigma_{-}, \\
& \frac{\widetilde{\mathbb{L}}_{0}^{2}}{\sqrt{h}} \stackrel{h \rightarrow 0}{\longrightarrow} L_{0}^{2}=\sqrt{\beta_{0}} \sigma_{+},
\end{aligned}
$$

and the other terms converges to 0 when $h$ tends to 0 . Thus the solution $\left(\widetilde{u}_{n}\right)_{n \in \mathbb{N}}$ of the equation

$$
\left\{\begin{array}{l}
\widetilde{u}_{n+1}=\widetilde{\mathbb{L}}_{n+1} \widetilde{u}_{n} \\
\widetilde{u}_{0}=I
\end{array}\right.
$$

is made of invertible operators which are locally uniformely bounded in norm and in particular $\widetilde{u}_{[t / h]}$ converges weakly to the solution $\widetilde{U}(t)$ of the equation

$$
\left\{\begin{array}{l}
d \widetilde{U}(t)=\left\{-\left(i \sigma_{z}+i \beta_{1} I+\frac{1}{2} \beta_{0} \sigma_{+} \sigma_{-}+\frac{1}{2} \beta_{1} \sigma_{-} \sigma_{+}\right) d t-i \sigma_{-}\left(\sqrt{\beta_{1}} d a_{0}^{1}(t)+\sqrt{\beta_{0}} d a_{2}^{0}(t)\right)\right. \\
\left.\quad-i \sigma_{+}\left(\sqrt{\beta_{1}} d a_{1}^{0}(t)+\sqrt{\beta_{0}} d a_{0}^{2}(t)\right)\right\} \widetilde{U}(t) \\
\widetilde{U}(0)=I .
\end{array}\right.
$$

Theorem 7.3 The quantum dynamical semigoup of the repeated quantum interaction model associated to the spin-boson system converges towards the equilibrium.

Proof For proving this theorem, it is suffices to see that the associated Lindbladian of the above equation is of the form

$$
\begin{aligned}
\mathcal{L}(X)=i\left[\sigma_{z}, X\right] & +\frac{1}{2} \beta_{0}\left[2 \sigma_{-} X \sigma_{+}-\left\{n_{-}, X\right\}\right] \\
& +\frac{1}{2} \beta_{1}\left[2 \sigma_{+} X \sigma_{-}-\left\{n_{+}, X\right\}\right] .
\end{aligned}
$$




\section{Remark}

Note that by using the repeated quantum interaction model we can prove that the Markovian properties of the spin-boson system are satisfied without using any assumption.

\section{References}

[AK] L. Accardi, S. Kozyrev: Quantum interacting particle systems. Volterra International School.

[AFL] L. Accardi, A. Frigerio, Y.G. Lu: Weak coupling limit as a quantum functional central limit theorem. Com. Math. Phys. 131, 537-570 (1990).

[ALV] L. Accardi, Y.G. Lu, I. Volovich: Quantum theory and its stochastic limit. SpringerVerlag Berlin, 2002.

[AL] R. Alicki, K. Lendi: Quantum dynamical semigroups and applications. Lecture Notes in physics, 286. Springer-Verlag Berlin, 1987.

[AJ] S. Attal, A. Joye: The Langevin Equation for a Quantum Heat Bath. J. Func. Analysis, to appear.

[AP] S. Attal, Y. Pautrat: From Repeated to Continuous Quantum Interactions. Annales Institut Henri Poincaré, to appear.

[B] A. Barchielli: Continual Measurements in Quantum Mechanics. Quantum Open systems. Vol III: Recent developments. Springer Verlag, Lecture Notes in Mathematics, 1882 (2006).

[BR] O. Bratteli, D.W. Robinson: Operator algebras and Quantum Statiscal Mechanics II, Volume 2. Springer-Verlag New York Berlin Heidelberg London Paris Tokyo, second edition 1996.

[C] R. Carbone: Optimal Log-Sobolev Inequality and Hypercontractivity for positive semigroups on $M_{2}(\mathbb{C})$, Infinite Dimensional Analysis, Quantum Probability and Related Topics, Vol. 7, No. 3 (2004) 317-335.

[D1] E.B. Davies: Markovian Master equations. Comm. Math. Phys. 39 (1974), 91-110.

[D2] E.B. Davies: Markovian Master Equations II.Math. Ann. 219 (1976), 147-158.

[D3] E.B. Davies: One-Parameter Semigroups. Academic Press London New York Toronto Sydney San Francisco, 1980.

[D4] E.B. Davies: Quantum Theory of Open Systems. Academic Press, New York and London, (1976).

[DJ] J. Derezinski, V. Jaksic: Return to Equilibrium for Pauli-Fierz Systems. Annales Institut Henri Poincaré 4 (2003), 739-793. 
[DJP] J. Derezinski, V. Jaksic, C.A. Pillet: Perturbation theory of $\mathrm{W}^{*}$-dynamics, KMS-states and Liouvillean, Rev. Math. Phys. 15 (2003), 447-489.

[DF] J. Derezinski, R. Fruboes : Fermi Golden Rule and Open Quantum Systems, Quantum Open systems. Vol III: Recent developments. Springer Verlag, Lecture Notes in Mathematics, 1882 (2006).

[F1] F. Fagnola: Quantum Stochastic Differential Equations and Dilation of Completely Positive Semigroups. Quantum Open systems. Vol II: The Markovian approach. Springer Verlag, Lecture Notes in Mathematics, 1881 (2006).

[F2] F. Fagnola: Quantum Markovian Semigroups and Quantum Flows. Proyecciones, Journal of Math. 18, n.3 (1999) 1-144

[F3] F. Fagnola: Characterization of Isometric and Unitary Weakly Differentiable Cocycles in Fock space. Quantum Probability and Related Topics VIII 143 (1993)

[FR1] F. Fagnola, R. Rebolledo: Nets of the Qualitative bihaviour of Quantum Markov Semigroups. Quantum Open systems. Vol III: Recent developments. Springer Verlag, Lecture Notes in Mathematics, 1882 (2006).

[FR2] F. Fagnola, R. Rebolledo: The Approach to equilibrium of a class of quantum dynamical semigroups. Inf. Q. Prob. and Rel. Topics, 1(4), 1-12, 1998.

[HP] R.L Hudson, K.R. Parthasarathy: Quantum Ito's formula and stochastic evolutions, Comm. Math. Phys. 93 (1984), no 3, pp.301-323.

[G] M. Gregoratti: The Hamiltonian Operator Associated with Some quantum Stochastic Evolutions Com. Math. Phys. 222, 181-200 (2001)

[JP1] V. Jaksic, C.A. Pillet: On a model for quantum friction II : Fermi's golden rule and dynamics at positive temperature. Comm. Math. Phys. 178, 627 (1996).

[JP2] V. Jaksic, C.A. Pillet: On a model for quantum friction III: Ergodic properties of the spin-boson system. Comm. Math. Phys. 178, 627 (1996).

[M] P. A. Meyer: Quantum Probability for Probabilists . Second edition. Lect Not. Math. 1538, Berlin: Springer-Verlag 1995.

[P] K. R. Parthasarathy: An Introduction to Quantum Stochastic Calculus. Birkhäuser Verlag: Basel. Boston. Berlin.

[R] R. Rebolledo: Complete Positivity and Open Quantum Systems. Quantum Open systems. Vol II: The Markovian approach. Springer Verlag, Lecture Notes in Mathematics, 1881 (2006). 